\title{
Reinhard Marx Folter: eine zulässige polizeiliche Präventionsmaßnahme?
}

\section{A. Einfübrung}

Der interne Aktenvermerk vom 1. Oktober 2002 des derzeit vom Dienst suspendierten Frankfurter Vizepolizeipräsidenten Wolfgang Daschner hat einen heftigen, nicht nur gesellschaftlichen, sondern insbesondere auch verfassungsrechtlichen Diskurs über die Zulässigkeit der Folter ausgelöst. Daschner hatte in dem Vermerk angeordnet, der der Kindesentführung verdächtige Magnus Gäfgen sei »nach vorheriger Androhung, unter ärztlicher Aufsicht, durch Zufügung von Schmerzen (keine Verletzung) erneut zu befragen. « Der vernehmende Polizeibeamte soll daraufhin dem Beschuldigten den Ernst der Lage verdeutlicht und darauf hingewiesen haben, dass » per Hubschrauber ein Experte eingeflogen werde, der ihm Schmerzen zufügen werde, wie er sie noch nie verspürt habe." Wenig später gab daraufhin Gäfgen den Aufenthaltsort des bereits toten elf Jahre alten Sohnes eines bekannten Frankfurter Bankiers bekannt. ${ }^{1}$ Zum ersten Mal in der Geschichte der Bundesrepublik Deutschland hat damit ein hoher verantwortlicher Polizeibeamter ihm untergebenen Beamten die Anweisung erteilt, Folter als Mittel der Aussagenerpressung anzuwenden. Nach Bekanntwerden des Aktenvermerks Mitte Februar 2003 wurde die Anordnung damit gerechtfertigt, polizeiliche Anwendung von Gewalt müsse erlaubt sein, wenn dadurch der unmittelbar bevorstehende Tod eines Kindes verhindert werden könne.

Die damit erstmals aus den theoretischen Debatten in die praktische Wirklichkeit überführte Figur der »Rettungsfolter ${ }^{2}$ macht deutlich, dass bisherige Gewissheiten über die absolute Schutzwirkung des völkerrechtlichen Folterverbotes in signifikanter Weise erschüttert worden sind. Insofern hat dieser Diskurs auch eine positive Wirkung, da er bewusst macht, dass allein die Berufung auf nationalsozialistische Verbrechen heute nicht mehr tragfähig ist, um Gefährdungen der Grund- und Menschenrechte abzuwehren. Wenn selbst die denkbare schärfste Verletzung der Menschenrechte, nämlich die Anwendung von oder die Drohung mit der Folter, als mögliches polizeiliches Instrument der Prävention in Betracht gezogen wird, dann muss diese Entwicklung die Menschenrechtsbewegung alarmieren. Sie muss aktiv in den Diskurs eingreifen, das Gespräch mit jenen führen, die im staatlichen Vollzug in der Verantwortung und verunsichert sind. Dabei kann sie nicht mehr davon ausgehen, dass bisherige Gewissheiten unhinterfragt dem Diskurs zugrunde gelegt werden können. Vielmehr muss sie angesichts der präventiven Begründung der Folteranwendung die für die Aufrechterhaltung des absoluten Folterverbotes maßgeblichen Grundsätze herausarbeiten und aktiv dafür werben, dass diese gesellschaftlich akzeptiert werden.

Nur vorübergehend dürfte die Atempause sein, welche die Bilder von $A b u$ Ghraib der Menschenrechtsbewegung verschaffen, weil andere bedeutende Ereignisse sehr schnell die öffentliche Aufregung in andere Richtungen lenken können. Die Suggestivkraft dieser die demütigende Fratze der Folter offenbarenden 

Bewusstsein für das absolute Folterverbot gestärkt und damit den Befürwortern der »Rettungsfolter« die Konstruktion ihrer dogmatischen und so schwer verdaulichen Abstraktionen erschwert; belegen sie doch, dass positivistische Versuche, die unbezähmbare Dynamik der Folter »rechtsstaatlich « einzufangen, an der hässlichen Wirklichkeit scheitern müssen. Andererseits geben die Bilder auch Kunde von einem erschreckenden Ausmaß an fehlendem Unrechtsbewusstsein von Angehörigen einer großen demokratischen Nation, die verabscheuungswürdiges, völkerrechtlich geächtetes Tun in Siegerpose verrichten. Die Menschenrechtsbewegung muss die Chance nutzen und die neu hervor gerufene Abscheu gegen die Folter strategisch aufgreifen, um Folterbegründungen im Rechtsstaat zu bekämpfen. Die Brechung des Willens der muslimischen Gefangenen scheint keinem anderen Zweck zu dienen als dem der auch sexistischen Lust an der Zerstörung ihrer menschlichen Würde. Doch bringen diese Bilder die gesamte Dynamik aus Unterwerfung, Erniedrigung und Entmannung auf den Punkt. Zwar geht es den Befürwortern der »Rettungsfolter « nach ihren Erklärungen um die Rettung von Menschenleben. So wie im Irak wollen sie die Folter nicht angewendet wissen. Vielmehr soll es »gesittet «, unter Beachtung des Grundsatzes der VerhältnismäBigkeit, zugehen. Es ist die Aufgabe der Menschenrechtsbewegung deutlich zu machen, dass es zwischen dem Fall Daschner und Abu Ghraib eine Verbindungslinie gibt. Dies legt ja bereits die sexistisch motivierte Drohung, Gäfgen mit zwei "großen Negern« in eine Zelle zusammen zu sperren, die sich an ihm sexuell vergehen könnten, nahe. Die Bewegung muss offensiv diskursive Strategien entwickeln, die überzeugungskräftig und plausibel in die demokratischen Gesellschaften hinein wirken und ein Bewusstsein dafür hervorrufen, dass staatlich angeordnete oder geduldete gezielte Schmerzzufügung nicht nur die Wahrheit hervorbringen will, sondern dieses Ziel nur erreichen kann, wenn sie die Persönlichkeit und die Psyche des Gefolterten zerstört. Alle geschichtlichen Erfahrungen belegen, dass mit der Zulassung der Folter der Kreislauf von Erniedrigung und Unterdrückung beginnt.

Die Chancen für eine erfolgreiche offensive Menschenrechtskampagne sind so schlecht nicht. International und auch in der Bundesrepublik hat sich seit 1945 eine selbstbewusste und von universellen Werten überzeugte Zivilgesellschaft heraus gebildet. Diese ist der Nährboden für die klare und von keinen Zweifeln hinsichtlich der absoluten Schutzwirkung des Folterverbotes geprägte internationale Rechtsprechung und insbesondere der des Europäischen Gerichtshofes für Menschenrechte in Straßburg. Der in den letzten fünf Jahrzehnten erworbene positivrechtliche Besitzstand ist ein hohes, in seiner überragenden Bedeutung für das gesellschaftliche Zusammenleben kaum zu überschätzendes Gut. Diese Rechtsprechung muss der Ausgangspunkt für eine menschenrechtliche Kampagne für die Stärkung der Menschenrechte, insbesondere des Folterverbotes, sein. Andererseits leben wir seit der Auflösung der globalen Blockkonfrontation 1989 in einer Zeit der allgemeinen Verunsicherung, die durch die terroristischen Aktionen des 11. Septembers 2001 noch um ein Vielfaches verstärkt wird. Der erworbene Besitzstand muss deshalb verteidigt werden und bedarf der Vergewisserung, in welcher Weise er angesichts der neuartigen Bedrohungen überzeugend und konsensstiftend legitimiert werden kann. Dazu bedarf es des offensiven Eintretens für die Grundwerte der universellen und der deutschen Verfassungsordnung und zugleich auch der Auseinandersetzung mit diesen neuartigen Bedrohungen.

Im Nachfolgenden werden deshalb zunächst aus Sicht des Völkerrechts die positivrechtlichen Grenzen polizeilicher Exmittlungs-und präventiver Arbeit darge- 
stellt. Anschließend erfolgt eine kritische Auseinandersetzung mit der neu geschaffenen Legitimationsfigur der »Rettungsfolter « und werden die Grundsätze heraus gearbeitet, die deren Legitimationsbasis in Frage stellen. Üblicherweise muss eine auf Überzeugung zielende Diskussion zunächst die Vor- und Nachteile einer umstrittenen Maßnahme aufzeigen, aber hinsichtlich deren Legitimation neutral bleiben. Es ist jedoch für eine menschenrechtlich motivierte Diskussion völlig undenkbar, dass in Ansehung der Folter im Ausgangspunkt eine neutrale Position eingenommen werden könnte. Vielmehr geht es darum, die von den Befürwortern der »Rettungsfolter « ins Feld geführten Argumente kritisch zu prüfen und insoweit wegen des Ziels der Konsensstiftung die insoweit gegebenen Begründungen nicht vorschnell abzuweisen, sondern ernsthaft zu prüfen.

\section{B. Das Verbot der Folter im Völkerrecht}

\section{Die Erfolgsgeschichte des Folterverbotes seit 1945}

Die nach 1945 verabschiedeten universellen und regionalen Menschenrechtsinstrumente untersagen die Anwendung der Folter in eindeutiger Weise. Nach Art. 5 der Allgemeinen Erklärung der Menschenrechte von 1948 (AEMR) darf "niemand der Folter oder grausamer, unmenschlicher oder erniedrigender Behandlung oder Strafe unterworfen werden «. Einen identischen oder ähnlichen Wortlaut enthalten Art. 7 des Internationalen Paktes über bürgerliche und politische Rechte von 1966 (IPbpR), Art. 3 der Europäischen Konvention zum Schutze der Menschenrechte und Grundfreiheiten von 1950 (EMRK), Art. 5 Abs. 2 der Amerikanischen Menschenrechtskonvention von 1969 sowie Art. 5 der (afrikanischen) Banjul Charta der Menschenrechte von 1981. Aufgrund dieser völkervertragsrechtlichen Entwicklung wird allgemein davon ausgegangen, dass das Folterverbot nicht nur den Rang einer völkergewohnheitsrechtlichen Norm erlangt hat, also auch Staaten bindet, die keinem der bezeichneten Verträge beigetreten sind, sondern darüber hinaus den Status eines ius cogens einnimmt. Es handelt sich damit um eine Norm, die von der internationalen Gemeinschaft in ihrer Gesamtheit angenommen und anerkannt wird, von der nicht abgewichen werden darf und die nur durch eine spätere Norm des allgemeinen Völkerrechts derselben Rechtsnatur geändert werden darf (Art. 53 Wiener Übereinkommen über das Recht der Verträge).

Darüber hinaus hat die Generalversammlung der Vereinten Nationen 1973 eine Erklärung und 1984 ein Übereinkommen gegen Folter und andere grausame, unmenschliche oder erniedrigende Behandlung oder Strafe verabschiedet. Dieses Übereinkommen hat die Bundesrepublik ratifiziert (BGBl. 1990 II S. 247). Es bindet deshalb die deutschen Behörden und erlegt der Bundesrepublik bestimmte Verpflichtungen auf, wirksame gesetzgeberische, verwaltungsmäßige, gerichtliche oder sonstige Maßnahmen zu treffen, um Folterungen in allen ihrer Hoheitsgewalt unterstehenden Gebieten zu verhindern (Art. 2 Abs. 1). Darüber hinaus ist der Gesetzgeber verpflichtet, alle Folterhandlungen strafrechtlich zu sanktionieren (Art. 4 Abs. 1). Die Bundesrepublik hat überdies Sorge dafür zu tragen, dass die Erteilung von Unterricht und die Aufklärung über das Verbot der Folter als vollgültiger Bestandteil in die Ausbildung des mit dem Gesetzesvollzug betrauten zivilen und militärischen Personals, des medizinischen Personals, der Angehörigen des öffentlichen Dienstes und anderer Personen aufgenommen wird, die mit dem Gewahrsam, der Vernehmung oder der Behandlung einer Person befasst werden 
können, die der Festnahme, der Haft, dem Strafvollzug oder irgendeiner anderen Form der Freiheitsentziehung unterworfen ist (Art. 10 Abs. 1). Im vertragstreuen Staat sind damit die Vollzugsbeamten über Inhalt, Reichweite und Schutzwirkung des Folterverbotes umfassend informiert, sodass im Falle der Zuwiderhandlung eine Berufung auf den schuldausschließenden Verbotsirrtum entfällt. Nach Art. 11 trifft die Bundesrepublik die Pflicht zur regelmäßigen systematischen Überprüfung der für Vernehmung geltenden Vorschriften, Methoden und Praktiken, um jeden Fall von Folter zu verhüten. Sobald ein hinreichender Grund für die Annahme besteht, dass im Bundesgebiet eine Folterhandlung begangen wurde, sind die zuständigen Behörden verpflichtet, umgehend eine unparteiische Untersuchung durchzuführen (Art. 12). Art. 15 enthält schließlich ein Beweisverwertungsverbot hinsichtlich von Aussagen, die durch Folter herbeigeführt worden sind.

Ein dichtes normatives Geflecht von Vorschriften stärkt damit das völkerrechtliche Folterverbot. Die hervorgebrachten Regelungen enthalten jedoch nicht nur lediglich bindende materielle Vorgaben für die Staatenpraxis. Vielmehr kennzeichnet die Erfolgsgeschichte des Folterverbotes darüber hinaus die Einrichtung internationaler Überprüfungsverfahren, um die Staatenpraxis zu überprüfen und auf ihre normativen Verpflichtungen festzulegen. Allgemein bekannt ist das Beschwerdeverfahren nach der Europäischen Menschenrechtskonvention, das nach Erschöpfung des innerstaatlichen Rechtsweges die Möglichkeit eröffnet, unmittelbar den Europäischen Gerichtshof für Menschenrechte anzurufen. Darüber hinaus hat der nach dem Europäischen Übereinkommen zur Verhütung von Folter von 1987 eingerichtete Ausschuss das Recht, periodische, aber auch ad hoc-Besuche in den Vertragsstaaten durchzuführen, um Berichten über Folterungen oder andere inhumane Maßnahmen nachzugehen und auf Abhilfe zu drängen. Dieses Verfahren ist nicht rechtsförmig, sondern präventiv gestaltet.

Auf der Ebene der Vereinten Nationen sind die Vertragsstaaten des Übereinkommens gegen Folter verpflichtet, dem Überwachungsorgan, also dem Ausschuss gegen Folter, in periodischen Abständen Staatenberichte vorzulegen, damit dieser die Einhaltung der Vorschriften des Übereinkommens überprüfen kann (Art. 19). Darüber hinaus kann der Ausschuss Untersuchungen durchführen, wenn er Hinweise auf systematische Folterungen in einem Vertragsstaat erhält, und geeignete Abhilfemaßnahmen vorschlagen (Art. 20). Schließlich kann sich ein Folteropfer nach Erschöpfung des innerstaatlichen Rechtsweges an den Ausschuss wenden, sofern der Vertragsstaat, gegen den sich die Beschwerde richtet, das individuelle Beschwerdeverfahren durch eine Erklärung anerkannt hat (Art. 21 Abs. 1 Satz 1). Der Ausschuss kann in diesem Zusammenhang seine guten Dienste zur Verfügung stellen, um eine gütliche Regelung der Beschwerde herbeizuführen (Art.21 Abs. 1 Buchst.e). Bedeutend effizienter als dieses schwerfällige rechtsförmige Verfahren ist das Besuchssystem, das nach europäischem Vorbild mit dem Fakultativprotokoll zum Übereinkommen gegen Folter vom 18. Dezember 2002 eingeführt worden ist, das bislang aber noch nicht in Kraft getreten ist. Es verpflichtet die beitretenden Staaten u. a., in allen Einrichtungen, in denen Menschen die Freiheit aufgrund behördlicher oder gerichtlicher Entscheidung entzogen ist, unabhängige Kontroll- und Beschwerdeinstanzen einzuführen. ${ }^{3}$ Bis Ende 2003 hatten das Fakultativprotokoll lediglich drei Staaten ratifiziert. Für das Inkrafttreten bedarf es jedoch der Ratifikation durch 20 Staaten. Auch die Bundesrepublik hat wohl wegen der bezeichneten Verpflichtung noch nicht ratifiziert. Darüber hinaus 
sind die präventiven Instrumente des Menschenrechtszentrums der Vereinten Nationen in Genf zu nennen. So berichtet z. B. der Sonderberichterstatter gegen Folter jährlich der Menschenrechtskommission über besonders besorgniserregende Folterpraktiken. In diesem Zusammenhang sind auch die anderen Sonderberichterstatter bzw. Arbeitsgruppen zu erwähnen, die sich mit besonders gravierenden Formen von Menschenrechtsverletzungen, insbesondere gegen inhaftierte Personen befassen.

Dieser geraffte Überblick über die Entwicklung des völkerrechtlichen Folterverbotes macht deutlich, dass das Verbot der Folter auf der normativen Ebene inzwischen eine kaum zu überschätzende Entwicklung erfahren hat, die noch dadurch verstärkt wird, dass dieses Verbot notstandsfest ist, also anders als andere Normen der menschenrechtlichen Verträge auch im Notstand, in Kriegszeiten oder zur Abwehr terroristischer Gefahren nicht eingeschränkt werden darf. Beispielhaft ist hier Art. 15 EMRK, demzufolge bestimmte Garantien eingeschränkt werden können, wenn das »Leben der Nation durch Krieg oder einen anderen öffentlichen Notstand bedroht ist«. Jedoch darf » in keinem Fall « vom Folterverbot des Art. 3 EMRK abgewichen werden (Art. 15 Abs. 2 EMRK). Auch nach Art. 4 Abs. 2 IPbpR ist das Folterverbot des Art. 7 IPbpR notstandsfest. Die anderen regionalen Instrumente enthalten identische Regelungen.

Andererseits ist nicht zu übersehen, dass nach 1945 die Praxis der Folter in vielen Staaten systematisch ausgeübt wird und weit verbreitet ist. Zwar hat die Zahl autoritärer Staaten abgenommen, wie etwa die Veränderungen in Chile und Osteuropa erweisen. Andererseits sind seit 1989 infolge der Veränderungen der Weltkarte und der in vielen Regionen zerfallenden Staaten neue Formen der Folterpraxis durch neue Akteure hinzugekommen, die, anders als Staaten, kaum oder nur unzulänglich mit überkommenen völkerrechtlichen Instrumenten angesprochen werden können. Hier versagen die rechtsförmigen Schutzinstrumente vollständig. Diplomatische oder andere politische Maßnahmen setzen eher selektiv an und sind kaum effektiv. Zwischen Norm und Praxis herrscht damit eine beträchtliche Kluft. Es wäre jedoch verfehlt, unter Hinweis auf die rechtswidrige Praxis das Insistieren auf die Herrschaft des Rechts aufzugeben und Verletzungen des Folterverbotes deshalb zu rechtfertigen. Recht war und ist stets mehr als bloße Praxis. Gerade wegen der aufgezeigten Kluft stellt sich die Herausforderung, die Geltungskraft der Norm in Inhalt, Umfang und Reichweite zu stärken und für den gesellschaftlichen Konsens zu werben.

\section{Völkerrechtliche Abgrenzungsfragen}

\section{Die Stigmatisierungsfunktion des Folterbegriffs}

Der notwendige gesellschaftliche Diskurs über die Reichweite der Schutzwirkung des absoluten Folterverbotes setzt eine klare juristische Begrifflichkeit voraus. Das Erfordernis begrifflicher Abgrenzung verdeutlicht insbesondere die DaschnerDebatte. So behaupten die Befürworter der »Rettungsfolter «, der interne Aktenvermerk vom 1. Oktober 2002 erfülle nicht die Voraussetzungen des Folterbegriffs. Dieser Rechtfertigungsversuch ist verständlich, hat doch der Europäische Gerichtshof für Menschenrechte darauf hingewiesen, dass die Verfasser der Europäischen Menschenrechtskonvention mit der Unterscheidung zwischen Folter und anderen unmenschlichen Maßnahmen dem Folterbegriff ein spezifisches 
Stigma anheften wollten. ${ }^{4}$ Generell ist anzumerken, dass man die hervorgebrachte

Schutzwirkung bestimmter Instrumente nicht durch unnötige Begriffsausweitungen schwächen sollte. Zwar verbietet Art. 3 der Konvention beide Maßnahmen, aber ein Staat, dem eine Folterpraxis nachgewiesen wird, verliert im internationalen Ansehen dramatisch an Reputation. Deshalb ist es verständlich, wenn jene, die aus nachvollziehbaren und verstehbaren Motiven polizeilichen Zwang zur Aussagenerpressung befürworten, sich dagegen wehren, mit Folterstaaten auf eine Stufe gestellt zu werden. Dies verdeutlicht, wie zentral gerade in der Debatte um die »Rettungsfolter« juristische Abgrenzungsfragen sind.

\section{Begriff der Folter}

Ausgangspunkt für die juristische Erfassung des Folterbegriffs ist Art. 1 Abs. 1 des Übereinkommens gegen Folter, der insoweit unter Rückgriff auf die bis dahin entwickelte Rechtsprechung und den wissenschaftlichen Diskurs Folter als jede Handlung definiert, »durch die einer Person vorsätzlich große körperliche oder seelische Schmerzen zugefügt werden, zum Beispiel, um von ihr oder einem Dritten eine Aussage oder ein Geständnis zu erlangen, um sie für eine tatsächlich oder mutmaßlich von ihr oder einem Dritten begangene Tat zu bestrafen oder um sie oder einen Dritten einzuschüchtern oder zu nötigen, oder aus einem anderen, auf irgendeiner Art von Diskriminierung beruhenden Grund, wenn diese Schmerzen oder Leiden von einem Angehörigen des öffentlichen Dienstes oder einer anderen in amtlicher Eigenschaft handelnden Person, auf seine Veranlassung oder mit deren ausdrücklichem oder stillschweigendem Einverständnis verursacht werden.« Es sind damit vier tatbestandliche Elemente, die den Folterbegriff prägen: Es muss eine dem Staat zurechenbare Handlung festgestellt werden, die Schmerzzufügung muss einen bestimmten Intensitätsgrad erreichen, die Handlung muss vorsätzlich begangen werden, und sie muss einen bestimmten Zweck verfolgen.

\section{a) Verantwortlichkeit des Staates für die Folterhandlung}

Nach der Definition des Übereinkommens muss die Folterhandlung dem Staat zurechenbar sein. Die Schmerzzufügung durch private Personen, die weder auf Veranlassung des Staates noch mit dessen ausdrücklichem oder stillschweigendem Einverständnis erfolgt, erfüllt danach nicht den Folterbegriff. Der Ausschuss gegen Folter hat allerdings in einem Flüchtlingsfall im Fall Somalia die dort agierenden »Warlords « unter den Begriff »Angehörige des öffentlichen Dienstes« (»public officials«) subsumiert, weil er insoweit den Begriff der »quasi-governmental institution « als erfüllt angesehen hat. ${ }^{5}$ Demgegenüber hat der Europäische Gerichtshof in ständiger Rechtsprechung einen deutlich weitergehenden Begriff der Regierungsverantwortlichkeit entwickelt. Danach ist der Staat positiv verpflichtet, Misshandlungen durch private Täter zu unterbinden. ${ }^{6}$ Ebenso wie der Ausschuss gegen Folter hält der Gerichtshof in Flüchtlingsfällen auch Übergriffe 
durch somalische Warlords für erheblich. ${ }^{7}$ Gleichwohl besteht zwischen beiden Ansätzen ein Unterschied, weil das Übereinkommen den Zurechnungsbegriff sehr eng fasst und damit Folterungen durch private Täter aus dem Folterbegriff herausfallen. Konzeptionell gelingt es demgegenüber dem Gerichtshof, auch Misshandlungen durch Private in den Folterbegriff zu integrieren. Allerdings sind bislang keine Fälle bekannt geworden, in denen Gewalt durch private Täter als dem Staat zurechenbare Folterhandlungen bewertet, wohl aber als unmenschliche Behandlung dem untätigen Staat zugerechnet wurden. ${ }^{8}$

\section{b) Grad der Schmerzzufügung}

Nach Art. 1 Abs. 1 des Übereinkommens setzt der Folterbegriff die Zufügung "großer körperlicher oder seelischer Schmerzen « voraus. Ebenso hat der Gerichtshof in ständiger Rechtsprechung den Unterschied zwischen Folterungen und anderen unmenschlichen oder erniedrigenden Behandlungen im Grad der Intensität der Schmerzzufügung gesehen. Während Foltermethoden, ${ }^{9}$ wie etwa die Palästinenserschankel ${ }^{10}$ oder Vergewaltigungen ${ }^{11}$ unzweifelhaft den erforderlichen Schweregrad erreichen, ist die erforderliche Abgrenzung in anderen Fällen nicht ohne weiteres derart eindeutig zu vollziehen. Diese ist nach Ansicht des Gerichtshofes notwendigerweise eine relative. Sie sei abhängig von allen Umständen des konkreten Einzelfalles, z. B. der Dauer der Behandlung, den körperlichen oder seelischen Auswirkungen, und in einigen Fällen dem Geschlecht, Alter und dem gesundheitlichen Zustand des Opfers. ${ }^{12}$ In diesem Zusammenhang herrschte im Nordirlandfall zwischen der (seit 1999 nicht mehr tätigen) Kommission und dem Gerichtshof Dissens in der Bewertung der in den Internierungslagern gegen Inhaftierte angewendeten fünf Vernehmungstechniken (stundenlanges an der Wandstehen, Überstülpen einer Kapuze über den Kopf des Vernehmenden, fortwährendes hohes zischendes Geräusch während der Vernehmung, Schlaf- und Nahrungsentzug). Während die Kommission wegen des Zusammenwirkens dieser fünf Techniken die für Folterhandlungen erforderliche Schwelle bejahte, wurde diese vom Gerichtshof verneint. ${ }^{13}$ Konnte damit früher der Relativitätstest dahin verstanden werden, als sei es im Falle polizeilicher und Schmerz zufügender Ermittlungsmethoden von den bezeichneten Kriterien abhängig, ob der erforderliche Grad der Schmerzzufügung erreicht worden sei, ist der Gerichtshof in seiner späteren Rechtsprechung dieser Interpretation entschieden entgegen getreten.

So hatte die französische Regierung im Falle eines in Polizeihaft misshandelten Anhängers einer korsischen Befreiungsorganisation argumentiert, wegen des jugendlichen Alters und des guten Gesundheitszustandes des Beschwerdeführers in Verbindung mit der Tatsache, dass dieser verdächtigt worden sei, an terroristischen Aktionen beteiligt gewesen zu sein, sei der erforderliche Schweregrad der Schmerzzufügung nicht erreicht worden. Der Gerichtshof wies diesen Einwand

7 ECHR, Abmed v. Austria, Reports 1996-VI, $₫ 44$.

8 ECHR, Cyprus v. Turkey, RJD 2001-IV, \$81; ECHR, Tyrer v. UK, Series A 26 \$29-35 (1978) - Körperstrafen, s. aber ECHR, Campbell and Cosans v. UK, HRLJ 1982, 221 (225); ECHR, $X$ and $Y v$. UK, HRLJ 1991, 61 (62); ECHR, Costello-Roberts v. UK, Series A 247-C (1993), in sämtlichen Fällen erreicht die Prügelstrafe in der Schule nicht die für unmenschliche Behandlung erforderliche Schwelle der Schmerzzufügung.

9 Christoph Grabenwarter, Europäische Menschenrechtskonvention 2003, S. 161.

10 ECHR, Aksoy v. Turkey, HRLJ 1997, 221 (227f.).

11 ECHR, Aydin v. Turkey, HRLJ 1998, 59 (68).

12 ECHR, Selmouni v. France, RID 1999-V = HRLj 1999, 238.

13 ECHR, Irland v. UK, Series A 25, \167(1978). 
deutlich zurück und verwies auf die ärztlich bestätigten, zahlreichen körperlichen

Zeichen von Gewalteinwirkung und deren Intensität. Darüber hinaus könnte die Notwendigkeit polizeilicher Ermittlungen zwecks Abwehr terroristischer Übergriffe nicht dazu führen, dass der konventionsrechtliche Schutz der körperlichen Unversehrtheit eingeschränkt werde. ${ }^{14}$ Polizeiliche Misshandlungen im Rahmen von Ermittlungen, die dem Opfer gezielt Schmerzen zufügen, erreichen damit stets den erforderlichen Schweregrad der Folterhandlung. Der Relativitätstest verfolgt vielmehr den Zweck, etwa bei Beschwerden über Haftbedingungen, körperliche Untersuchungen, polizeiliche Bedrohungen und rassische Diskriminierungen die Abgrenzung zwischen Folterhandlungen und anderen unmenschlichen oder erniedrigenden Behandlungen vorzunehmen.

Der Gerichtshof hat darüber hinaus in Übereinstimmung mit seiner Auffassung, dass die Konvention dem Wandel der Rechtsauffassung Rechnung tragen müsse, also ein »living instrument « ${ }^{15}$ sei, hervorgehoben, dass bestimmte Maßnahmen, die in der Vergangenheit lediglich als »inhuman und erniedrigend « klassifiziert worden seien, in Zukunft als Folterhandlungen bewertet werden könnten. Der zunehmende hohe Standard, der im Bereich des Menschenrechtsschutzes zu beachten sei, erfordere größere Nachdrücklichkeit bei der Feststellung von Verletzungen fundamentaler Werte demokratischer Gesellschaften. Deshalb bewertete der Gerichtshof die polizeilichen Misshandlungen eines marokkanischen Beschwerdeführers in französischer Polizeihaft als Folter. Der Beschwerdeführer war gezwungen worden, durch ein Spalier von Polizeibeamten zu laufen, und war dabei geschlagen worden. Er hatte sich vor einer jungen Frau hinknien müssen, zu der ein Beamter sagte, »Schau, du wirst gleich jemand singen hören.« Ein anderer Polizeibeamter hatte ihm seinen Penis gezeigt und gedroht: "Schau, lutsch dies «, und hatte anschließend über seinen Körper uriniert. Schließlich war er mit einer Lötlampe und einer Spritze bedroht worden. Der Gerichtshof verwies auf die Vielzahl der inhumanen Handlungen und stellte fest, dass diese, unabhängig von deren gewaltsamer Natur, für jedermann abscheulich und erniedrigend seien, unabhängig von ihren Bedingungen. Betrachte man die angewendete körperliche und seelische Gewalt als Ganzes, hätte sie dem Beschwerdeführer ernsthafte Schmerzen zugefügt und sei sie insbesondere ernsthaft und grausam gewesen. Eine derartige Behandlung müsse als Folter bezeichnet werden. ${ }^{16}$

Bewertet man anhand der Rechtsprechung des Gerichtshofes den internen Aktenvermerk und dessen Umsetzung, dürfte der erforderliche Grad der Schmerzzufügung erreicht worden sein. Die Anweisung schloss zwar ausdrücklich aus, dass Verletzungen zugefügt werden. Daschner hat nach Bekanntwerden der Anweisung nochmals hervorgehoben, dass "Schlagen, Zufügen von Verletzungen, Einsatz von Hilfsmitteln « von ihm ausdrücklich ausgeschlossen worden seien. Auf Frage, was geschehen wäre, hätte Gäfgen trotz Gewaltanwendung nicht mehr geschwiegen, hatte er geantwortet: »Irgendwann hätte er nicht mehr geschwiegen «. Daschner wies darauf hin, dass es sich bei einem solchen Vorgehen um »eine polizeiliche Maßnahme« und nicht etwa um eine Folterhandlung gehandelt habe. Dem ist zu widersprechen. Für die völkerrechtliche Folterdefinition ist es unerheblich, dass Verletzungen ausgeschlossen wurden. Denn es kommt nicht auf Verletzungen, sondern auf die Schmerzzufügung an. In Ausführung des Aktenvermerks hatte ein Polizeibeamter Gäfgen gedroht, es werde ihm Schmerzen zugefügt werden, wie er sie noch nie verspürt habe. Per Hubschrauber werde ein 
Experte eingeflogen, der diese Schmerzen zufügen werde. Dabei habe der Beamte das Geräusch eines Hubschraubers imitiert und überdies gedroht, Gäfgen werde »mit zwei großen Negern in eine Zelle gesperrt «, welche sich an ihm »sexuell vergehen könnten. Daschner hatte in einem Zeitungsinterview bestätigt, dass ein Polizeibeamter, der eine Übungsleiterlizenz des Deutschen Sportbundes besitzt und sich damals gerade im Urlaub befand, eingeflogen werden sollte, um Gäfgen Schmerzen zuzufügen.

Für die Frage, ob der Aktenvermerk und seine Ausführung den erforderlichen Grad der Schmerzzufügung erreicht hat, kommt es auf eine Gesamtbewertung der Maßnahmen an. Bereits die Drohung mit einem empfindlichen Übel in Verbindung mit vergleichbaren inhumanen und erniedrigenden Maßnahmen kann den erforderlichen Grad der Schmerzzufügung erreichen. Ob man den Aktenvermerk nach innerstaatlichen strafrechtlichen Grundsätzen lediglich als Versuch der Aussagenerpressung werten will oder nicht, ist für die völkerrechtliche Bewertung unerheblich. Insoweit hat der vernehmende Polizeibeamte auf Veranlassung seines Vorgesetzten (vgl. Art. 1 Abs. 1 Satz 1 des Übereinkommens gegen Folter) eine Vielzahl von Bedrohungen ausgesprochen, die in ihrer Gesamtbewertung durchaus den erforderlichen Grad der Schmerzzufügung erreicht hatten. Dies wird auch daraus ersichtlich, dass Gäfgen zwecks Vermeidung deren Realisierung eine Aussage gemacht hatte. Daschner hatte in dem bezeichneten Zeitungsinterview ausgeführt, es gebe »die Möglichkeit, durch einfache körperliche Einwirkung, z. B. durch Überdehnen eines Handgelenkes, Schmerzen zuzufügen. Es gibt am Ohr bestimmte Stellen - jeder Kampfsportler weiß das -, wo man draufdrückt, und es tut sehr weh, ohne dass irgendeine Verletzung entsteht. « ${ }^{17}$ Sofern man auch bei einer Gesamtschau der Bedrohungen gegen Gäfgen diese nicht als ausreichend ansehen mag, ist festzuhalten, dass diese jedenfalls als unmenschliche Behandlung und damit als völkerrechtlich unzulässig einzustufen sind. Daschner hatte eine klare Anweisung zur Schmerzzufügung gegeben. Jedenfalls dann, wenn die von ihm intendierte körperliche Gewalt durch den eingeflogenen »Experten « realisiert worden wäre, wäre dem Betroffenen zweifellos ein als Folter zu bewertender Schmerz zugefügt worden.

\section{c) Vorsätzliche Handlung}

Nach Art. 1 Abs. 1 des Übereinkommens gegen Folter muss die in Rede stehende Handlung vorsätzlich verübt werden. Ebenso verlangt der Gerichtshof für die Klassifizierung einer Handlung als Folter deren vorsätzliche Begehung. ${ }^{18}$ Demgegenüber wird bei den unmenschlichen oder erniedrigenden Behandlungen - wie etwa das Beispiel der Haftbedingungen erweist - keine vorsätzliche Begehungsform gefordert. Zwar zieht der Gerichtshof selbst nicht absichtlich verursachte Gefahren für Leib und Leben in Betracht. So hat er die dramatische Verschlechterung des Gesundheitszustandes infolge von Abschiebung im Rahmen von Art. 3 EMRK berücksichtigt. ${ }^{19}$ Anknüpfungspunkt für das vorsätzliche Handeln ist in diesem Zusammenhang aber der die Abschiebung durchführende Vertragsstaat. Dieser muss vor der Abschiebung alle erkennbaren oder vorgetragenen konkreten Risiken für die körperliche Unversehrtheit des Beschwerdeführers im Zielstaat der Abschiebung, unabhängig davon, durch wen sie verursacht werden, be- 
rücksichtigen und ausschließen. Kann er dies nicht und führt er gleichwohl die Abschiebung durch, verletzt er vorsätzlich Art. 3 EMRK. ${ }^{20}$

\section{d) Zweckrichtung der Misshandlung}

Schließlich werden nur die Misshandlungen als Folter qualifiziert, die ausgeübt werden, um eine Aussage oder ein Geständnis zu erlangen, um den Misshandelten für eine tatsächlich oder mutmaßlich von ihm oder einem Dritten begangene Tat zu bestrafen, oder um diesen oder einen Dritten einzuschüchtern oder zu nötigen, oder aus einem anderen, auf irgendeiner Art von Diskriminierung beruhenden Grund (Art. 1 Abs. 1 Übereinkommen gegen Folter). Das Übereinkommen bezeichnet damit eine weite Bandbreite von Zweckrichtungen. Fehlt es an einem derartigen Zweck, liegt lediglich eine unmenschliche Behandlung oder Strafe vor. Umgekehrt wird keine Folter angenommen, wenn zwar bestimmte zweckgerichtete Misshandlungen festgestellt werden, die Misshandlungen als solche jedoch nicht die erforderliche Schwere aufweisen. ${ }^{21}$

\section{C. »Rettungsfolter« im Lichte des Völkerrechts}

\section{Eine newartige Theorie der »Güterabwägung»}

In einer Reihe von Zeitungsmeldungen im Februar 2003 wurde Daschner mit der Forderung zitiert, der Einsatz von Gewalt bei Behörden müsse als letztes Mittel rechtlich zulässig sein, um Menschenleben zu retten. Der Vorfall löste eine heftige Debatte über die Frage aus, ob es Situationen geben könne, in denen in Deutschland Folterhandlungen zulässig sein sollten. Während viele Politiker und Vertreter der Zivilgesellschaft derartigen Überlegungen unverzüglich entgegentraten, bekundeten insbesondere regionale Politiker und andere Personen des öffentlichen Lebens Verständnis für Daschner und erklärten, sie könnten sich in Deutschland Ausnahmen vom Folterverbot vorstellen. Eine Forsa-Umfrage für die Illustrierte "Stern« ergab, dass $63 \%$ der Befragten sich gegen eine strafrechtliche Verfolgung von Daschner aussprachen. Während aus aktuellem Anlass initiierte, komplexe Fragestellungen auf verkürzende Alternativen reduzierende demoskopische Umfragen noch keine verlässlichen Aussagen über gesellschaftliche Wertvorstellungen zulassen, wiegt schwerer, dass der Präsident des Bundesgerichtshofes zum Fall Daschner erklärte, dass zwar das Folterverbot ein »hohes ethisches, rechtliches und kulturelles Prinzip « sei, die strafrechtliche Prüfung des Einzelfalles damit aber nichts zu tun habe. ${ }^{22}$ Angesichts der Bedeutung, die dem Kampf gegen Straflosigkeit im Hinblick auf schwerwiegende Menschenrechtsverletzungen zukommt, stärken diese diffusen Windungen eines hohen deutschen Justizbeamten zumindest nicht das erforderliche Rechtsbewusstsein gegen die Folterpraxis

Der Aktenvermerk und seine praktische Ausführung stellen zwar einen besonders spektakulären Angriff auf das absolute Folterverbot dar und ist auch insoweit singulär, als bislang in Deutschland kein hoher Polizeibeamter nachhaltig und offensiv für Ausnahmen vom Folterverbot eingetreten ist. Der philosophische und 
verfassungsrechtliche Diskurs über diese Frage begann jedoch bereits Mitte der siebziger Jahre des vorigen Jahrhunderts. So hatte 1976 der damalige niedersächsische Ministerpräsident Ernst Albrecht in seiner Monographie »Der Staat. Idee und Wirklichkeit« die Auffassung vertreten, dass Folter zur Abwehr einer terroristischen Aktion sittlich geboten sein könne. Bemerkenswert ist, dass Albrecht diese Forderung nicht in dem Abschnitt, der den wirklichen Staat behandelt, sondern im Abschnitt über den idealen Staat erhob. ${ }^{23}$ Der als erwünscht vorgestellte ideale Staat wurde danach als Staat konzipiert, den unter bestimmten Voraussetzungen die Verpflichtung zur Folteranwendung trifft. In Reaktion auf eine durch amnesty international initiierte Kampagne gegen diese Forderung zog Albrecht diese zurück und brachte eine zweite Auflage seiner Monographie ohne die umstrittene Passage heraus. Bedeutsam ist dieser längst vergessene Vorfall gleichwohl, war doch der Autor immerhin ein führendes Mitglied der politischen Elite und 1980 sogar als Kandidat für das Amt des Bundeskanzlers im Gespräch.

Ein Vierteljahrhundert nach diesem Vorfall vertrat der Heidelberger Rechtsphilosoph und Verfassungsrechtler Winfried Brugger in der angesehenen, insbesondere den verfassungspolitischen Diskurs fördernden Juristenzeitung die These, dass die durch eine terroristische Aktion bedrohten Bürger gegen den von der Polizei festgenommenen und der Komplizenschaft mit den Tätern verdächtigten Mittäter ein subjektives Recht auf Folteranwendung hätten, wenn durch seine Aussage die terroristische Gefahr abgewendet werden könne. ${ }^{24}$ Danach kann also jeder gefährdete Bürger - und das ist bei terroristischen Bedrohungen heutigen Ausmaßes jeder Bürger - bei dem für den Gewahrsamsort zuständigen Verwaltungsgericht einen Antrag nach $\$ 123 \mathrm{VwGO}$ auf Erlass einer einstweiligen Anordnung stellen, um eine unwillige Polizei zu zwingen, gegen den Komplizen zwecks Aussagenerpressung die Folter anzuwenden. Bemerkenswert ist die Reaktion der Zunft der Verfassungsrechtler auf diese abwegige These: Sie schwieg. Brugger entwickelt eine »Schutznormlehre«, welche die Voraussetzungen festlegt, unter denen eine Handlungspflicht der Polizei zum Foltern sich zugleich in einen Anspruch der durch die Pflicht begünstigten Bürger wandelt. Er bestreitet, dass seine Lehre zu einer generellen Schwächung des Folterverbotes führen werde, sondern im Gegenteil »durch Spezifizierung und Herausnahme einer Fallgruppe, in der das absolute Folterverbot zu widersinnigen und ungerechten Ergebnissen, zu einem >ethischen Skandalon führen würde«, dieses stärken werde. Die absoluten Folterverbote seien bei terroristischen Gefahren erheblichen Ausmaßes »ungerecht«. Sie aufrechtzuerhalten, untergrabe das Vertrauen der Bürger in die Rechtsordnung als System gegenseitiger Sicherheit und Freiheit. Es gehe um eine »Rechtsabwägung und Spezifizierung der Ausnahmesituation«. Es stehe »körperliche Integrität gegen körperliche Integrität und Würde gegen Würde«. Und falls ein solcher Konflikt nicht anderweitig auflösbar sei, dürfe im Zweifel der Staat die Interessen des Opfers denjenigen der Täter überordnen. Brugger stellt eine Wertungslücke in der Methodenlebre fest, die er mit dem Grundsatz der effektiven Gefahrenabwehr zugunsten der »Rettungsfolter « schließen will. ${ }^{25}$ Die Menschenwürde komme jedem Menschen zu, auch dem Störer und vermutlichen Straftäter. Doch bleibe »hier schon ausgeblendet, dass der drohende Zwang nur dazu dienen soll, den Erpresser in den ihm zustehenden Rechtsraum zurückzudrängen, den er eigenmächtig und in Verletzung individueller Rechte anderer überschritten hat. 
Kantisch gesprochen, wird der Zwang nur ausgeübt, um den Freiheitsgebrauch aller wieder kompatibel zu machen. $\ll^{26}$

Diese zunächst vereinzelte verfassungsrechtliche Position hat mit dem Erscheinen der 42. Ergänzungslieferung des führenden Kommentars zum Grundgesetz im Februar 2003 eine bislang noch nicht absehbare, neuartige verfassungspolitische Dynamik erfahren. Die von Günter Dürig begründete Kommentierung zu Art. 1 Abs. 1 des Grundgesetzes, die über fünfzig Jahre den verfassungsrechtlichen Diskurs zur Menschenwürdegarantie und die Rechtsprechung des Bundesverfassungsgerichts geprägt hatte, wurde ersetzt durch die Neukommentierung des Bonner Verfassungsrechtlers Mattbias Herdegen. Dieser löst die absolute Würdegarantie im Interesse einer Abwägungsoffenheit auf. Ausdrücklich wendet er sich gegen die Interpretation der Menschenwürde als »unantastbare Garantie«. Seit den Beratungen des Parlamentarischen Rates habe die kategorische Ächtung bestimmter Formen des staatlichen Terrors das Verfassungsverständnis geprägt. Dieses Verständnis sperre sich gegen die »Öffnung des Würdegehalts und einer Diagnose der Würdeverletzung für irgendwelche Abwägungen.« Nur in wenigen Fällen, etwa beim Genozid oder bei Massenvertreibungen, gebe es jedoch Evidenzen für eine Verletzung des »Würdekerns". Jenseits dieses »engsten Kreises von Würdeverletzungen «, die durch »Verfolgungsmaßnahmen totalitärer Regime und polizeiliche Exzesse aus rassisch-ethnischen Gründen ausgefüllt « werde, müsse der Menschenwürde ein »Begriffshof « zugeordnet werden, der für eine bilanzierende Würdigung aller für die Schwere des Eingriffs und des verfolgten Zwecks maßgeblichen Umstände offen ist. Hierfür spricht, dass dem Würdeanspruch der Schutz vor völlig unangemessenen Eingriffen im Sinne eines rudimentären Übermaßverbotes immanent ist. Auf der Basis einer derartigen Abschichtung von Würdekern und weiterem Schutzbereich (Begriffshof) werde auch eine »sachgerechte Beurteilung körperlicher und seelischer Eingriffe für präventive Zwecke « erreicht. Die gängigen »Lehrbuchprobleme« der körperlichen Schmerzzufügung zur Rettung von Menschenleben würden verkürzt, »wenn jede Anwendung derart willensbeugender oder willenskontrollierender Eingriffe rein modal beurteilt und deshalb stets - in völliger Abstraktion vom intendierten Lebensschutz - als Würdeverletzung beurteilt wird.« Daraus könne sich im Einzelfall ergeben, »dass die Androhung oder Zufügung körperlichen Übels, die sonstige Überwindung willentlicher Steuerung oder die Ausforschung unwillkürlicher Vorgänge wegen der auf Lebensrettung gerichteten Finalität eben nicht den Würdeanspruch verletzen. ${ }^{27}$

Was Herdegen nicht vorhersehen konnte, hat Daschner materialisiert. Er hat die schwer verdauliche dogmatische Abstraktion Herdegens konkretisiert; vorgemacht, dass es nach seiner Auffassung nicht beim »Lehrbuchproblem «, bei der "geringen praktischen Relevanz unausweichlicher Konflikte « 28 bleiben darf. Der führende Standardkommentar zum Grundgesetz, an dem immerhin der frühere Präsident des Bundesverfassungsgerichtes und frühere Bundespräsident, Roman Herzog, sowie die führenden Mitglieder der Gesellschaft der Verfassungsinterpreten beteiligt sind, verwendet nunmehr für die inhaltliche Konkretisierung der verfassungsrechtlichen Menschenwürdegarantie die dogmatisch geschulten Juristen so bekannte Differenzierung in »Kerngehalt « und »Vorhof «, die insbesondere 
bei uneingeschränkt gewährleisteten Grundrechten angewendet wird. ${ }^{29}$ Der Kerngehalt ist abwägungsfeindlich, indes auf die Exzesse totalitärer Regime, den Genozid und Massenvertreibungen begrenzt, für die derzeitige Bundesrepublik mithin ohne Bedeutung. Unterhalb dieser extrem hohen Schwelle - und damit ausnahmslos für alle bei uns derzeit vorstellbaren Fallkonstellationen - wird das absolute Folterverbot des Völkerrechts jedenfalls mit Wirkung für die deutsche Verfassungswirklichkeit aufgelöst und einem »bilanzierenden « Abwägungsgebot anheim gegeben. Wie die willentlich freigelassenen Geister gebannt werden können, verrät der Autor nicht. Fatalistisch stellt er statt dessen fest, dass der Konsens über unverbrüchliche Werte leicht bei jedem konkreten Szenario zerbreche, an dem sich »ein abwägungsfreier Würdeschutz der Rettung von Menschenleben in den Weg zu stellen scheint.«

Der ehemalige Bundesverfassungsrichter und Verfassungslehrer Ernst-Wolfgang Bökkenförde erkennt in Herdegens Neukommentierung einen "Epochenwechsel«. Dürigs Kommentierung habe die Menschenwürdegarantie »als Übernahme eines grundlegenden, in der europäischen Geistesgeschichte hervorgetretenen >sittlichen Werts in das positive Verfassungsrecht« verstanden, »das sich dadurch selbst auf ein vorpositives Fundament, eine Art naturrechtlicher Anker « beziehe. Die Menschen würde sei nach Dürig - und vom Bundesverfassungsgericht so übernommen - dann getroffen, wenn der konkrete Mensch zum Objekt, zu einem bloßen Mittel, zur vertretbaren Größe herabgewürdigt werde. Demgegenüber stelle Herdegens Neukommentierung die Menschenwürde ganz auf sich, löse und schneide sie $\mathrm{ab}$ »von der Verknüpfung mit dem vorgelagerten geistig-ethischen Inhalt.« Was hierzu zu sagen sei, wandere ab in den »geistesgeschichtlichen Hintergrund «, worüber kundig berichtet werde, indes ohne normative Relevanz. Zutreffend spitzt Böckenförde seine Kritik zu: Was der Abfolge der Kommentierungen ihre symptomatische Bedeutung verleihe, sei die Veränderung der Argumentationsebene und des grundsätzlichen Ansatzpunktes. Es sei der »Wechsel im Verständnis der Menschenwürdegarantie vom tragenden Fundament der neu errichteten staatlichen Ordnung, das deren Identität aufweist, zu einer Verfassungsnorm auf gleicher Ebene neben anderen, die rein staatsrechtlich, das heißt, auch sich heraus positivrechtlich zu interpretieren ist. A Art. 1 Abs. 1 des Grundgesetzes sei »nicht mehr die Grundfeste und meta-positive Verankerung der grundgesetzlichen Ordnung, nicht mehr >Pfeiler im Strom< des verfassungsrechtlichen Diskurses, sondern fließt darin mit, anheim gegeben und anvertraut der Gesellschaft der Verfassungsinterpreten, für die kein verbindlicher Kanon der Interpretationswege existiert. « 30

Die Entwicklung des verfassungspolitischen Diskurses ist besorgniserregend. Das Grundgesetz enthält kein ausdrückliches Folterverbot. Es wird aus dem Zusammenhang der Vorschriften des Art. 1 Abs. 1 mit Art. 2 Abs. 2 Satz 1 verfassungsrechtlich konstruiert und durch das Verbot des Art. 104 Abs. 1 Satz 2 Grundgesetz, das die seelische und körperliche Misshandlung von Festgenommenen verbietet, bestätigt. Der dargestellte Diskurs verdeutlicht indes, wie schwach das Grundgesetz auf normativer, dogmatischer Ebene gegen Relativierungen unverbrüchlicher Werte im Interesse einer letztlich der Beliebigkeit anheim gegebenen Abwägungs- und Schaukellehre ist. Da Art. 104 Abs. 1 Satz 2 Grundgesetz dem Kerngehalt der Menschenwürdegarantie zugeordnet wird, wird seine Schutzwir- 

mittelbar ergriffen werden. Der bislang angelaufene verfassungspolitische Diskurs ist auf den deutschen Geltungsrahmen begrenzt. Die Rechtsprechung des Europäischen Gerichtshofes für Menschenrechte wird lediglich begrenzt und in verkürzender Weise ${ }^{31}$ rezipiert. Lediglich Böckenförde verweist auf die grundlegenden Werte der europäischen Geistesgeschichte, zu der nach der Rechtsprechung des Gerichtshofes insbesondere das abwägungsfeste, absolute Folterverbot gehört. Besorgniserregend erscheint insbesondere, dass ohne textliche Änderung der Verfassung - allein durch die Schar der Verfassungsinterpreten - das Folterverbot aufgelöst und einer Praxis der Weg in deutsche Amtsstuben eröffnet werden kann, die die bestehende Verfassungsordnung von innen heraus zersetzen würde.

Allein die »Gesellschaft der Verfassungsinterpreten « kann diese Kehrtwende nicht vollziehen, wenn nicht das Bundesverfassungsgericht den Diskurs aufgreift. Dieses zeigt sich jedoch bislang unbeeindruckt von der Neukommentierung und hält im Urteil vom 3. März 2004 zum Lauschangriff an seiner gefestigten Rechtsprechung fest: Die Menschenwürde ist oberstes Konstitutionsprinzip und oberster Verfassungswert. Anknüpfend an die Erfahrungen in der Zeit des Nationalsozialismus standen in der Rechtsprechung zunächst Erscheinungen wie Misshandlung, Verfolgung und Diskriminierung im Zentrum der Überlegungen. Später wurde die Menschenwürdegarantie im Hinblick auf neue Gefährdungen maßgebend, so in den 1980er Jahren für den Missbrauch der Erhebung und Verwertung von Daten. Gegenwärtig bestimmen insbesondere Fragen des Schutzes der personalen Identität und der psychisch-sozialen Integrität die Auseinandersetzungen über den Menschenwürdegehalt. Es ist mit der Würde des Menschen nicht vereinbar, ihn zum bloßen Objekt der Staatsgewalt zu machen. Allerdings sind der »Leistungskraft der Objektformel " auch Grenzen gesetzt. »Die Menschenwürde ist nicht schon dadurch verletzt, dass jemand zum Adressaten von Maßnahmen der Strafverfolgung wird, wohl aber dann, wenn durch die Art der ergriffenen Maßnahme die Subjektqualität des Betroffenen grundsätzlich in Frage gestellt wird. Das ist der Fall, wenn die Behandlung durch die öffentliche Gewalt die Achtung des Wertes vermissen lässt, der jedem Menschen um seiner selbst willen zukommt. Solche Maßnahmen dürfen auch nicht im Interesse der Effektivität der Strafrechtspflege und der Wabrheitserforschung vorgenommen werden. «32

\section{Eine Herausforderung für die Menschenrechtsbewegung}

Änderungen der bestehenden Gesellschafts- und Rechtsordnung und damit einhergehend inhaltliche Umwandlungen überkommener Werte erfolgen in aller Regel nicht eruptiv, schlagartig, mit der Folge, dass gegenläufige gesellschaftliche Mobilisierungsschübe ausgelöst werden. Vielmehr werden diese häufig durch eine Vielzahl von Ereignissen und Diskussionen bewirkt, die jeweils für sich genommen zunächst die Gemüter erhitzen, nach einer gewissen Zeitspanne aber wieder abflauen. Unmerklich und eher stillschweigend verändert die Abfolge der unterschiedlichen Vorfälle die Anschauungen über universelle und gesellschaftliche Werte. Es ist deshalb die Aufgabe der Menschenrechtsbewegung, mit dem Ohr ganz unmittelbar und nah am Puls der gesellschaftlichen Entwicklung zu bleiben, mit besonderer Sensibilität wie ein hochempfindlicher Seismograph Pulsausschlä- 
ge zu registrieren und im konkreten Fall Alarm zu schlagen, um diesem unmerklichen Prozess des Zerfalls der Wertvorstellungen entgegen zu wirken. Wie die Reaktionen auf den Aktenvermerk belegen, kann die Bewegung sich auf einen grundlegenden gesellschaftlichen Konsens, wie er in der höchstrichterlichen Rechtsprechung und in politischen Meinungsäußerungen der führenden Eliten zum Ausdruck kommt, berufen. Die Bezugnahme auf den positivrechtlichen Besitzstand ist damit eine zentrale Voraussetzung für die Entwicklung einer menschenrechtlichen Gegenstrategie gegen Erschütterungen des Folterverbots.

Wie jedoch die Diskussion um die deutsche Menschenwürdegarantie erweist, besteht die Gefahr, dass eine ausschließlich positivrechtliche Herangehensweise den herauf gezogenen und ziehenden Gefahren für die universelle Werteordnung nicht gerecht werden kann. Vielmehr müssen die zentralen Motive und Rechtfertigungen für das absolute Folterverbot vor dem Hintergrund neuartiger terroristischer Bedrohungen neu diskutiert werden. Die Befürworter der »Rettungsfolter « mobilisieren intensive, moralisch aufgeladene Stimmungsbilder, verweisen darauf, dass die handelnden Polizeibeamten vor Ort »nur die Wahl zwischen zwei (rechts-)fehlerhaften Verhaltensweisen« hätten. »Sich angesichts dieses im Wortsinne tragischen Dilemmas in den Schmollwinkel der moralisch-rechtlichen Gewissheit zurückzuziehen und die Entscheidungsträger dahingehend zu belehren, dass Eingriffe in die Menschenwürde unter keinen Umständen rechtfertigungsfähig seien, ist wohlfeil, aber spätestens seit den Frankfurter Vorfällen - sit venia verbo - verantwortungslos «. ${ }^{33}$ Damit wird deutlich, dass das Beharren auf überkommenen unverbrüchlichen universellen Werten als verantwortungslose Gesinnungsethik denunziert wird. Es muss deshalb dem Eindruck entgegen gewirkt werden, dass das Insistieren auf die Beachtung des absoluten Folterverbotes abstrakt bleibender Fundamentalismus ist, der ohne gute Gründe selbst den Tod unschuldiger Kinder um eines Prinzips willen in Kauf nimmt. Vielmehr ist die hier deutlich werdende dualistische Aufspaltung in Verantwortungsethik und Gesinnungsethik aufzubrechen und überzeugend zu vermitteln, dass der unbeirrbare Einsatz für das Folterverbot aus der Sorge um den Bestand der freiheitlichen Gesellschafts- und Staatsordnung seine Motivation erfährt

\section{Eine menschenrechtliche Strategie gegen Erschütterungen des Folterverbotes}

\section{Berufung auf völkerrechtliche Verpflichtungen}

Die Menschenrechtsbewegung ist international organisiert, legitimiert sich durch universelle Werte und beruft sich gegenüber Regierungen auf internationale Normen. Daher kann nicht das deutsche Grundgesetz und der in Deutschland hervorgebrachte Konsens über den Achtungsanspruch der Menschenwürdegarantie die Basis für die Entwicklung einer menschenrechtlichen Strategie sein. Andererseits muss eine auf die deutsche Gesellschaft einwirkende Strategie den verfassungsrechtlichen und -politischen Diskurs integrieren, das heißt, diesen berücksichtigen, wenn er den Achtungsanspruch völkerrechtlicher Normen verstärkt, und entschieden zurück weisen, wenn er mit universellen Werten in Widerspruch gerät. Für das absolute Folterverbot hat dies zur Folge, dass die Menschenrechtsbewegung sich vorrangig auf dessen ius cogens-Charakter beruft. Anders als die 
deutsche Verfassung, die auf eine naturrechtliche Legitimation zurück greifen muss, um die uneingeschränkte Geltung des Folterverbots als Ausfluss der Menschenwürdegarantie zu begründen, erfolgt die völkerrechtliche Begründung der absoluten Schutzwirkung dagegen positivrechtlich.

Der Europäische Gerichtshof für Menschenrechte weist in ständiger Rechtsprechung darauf hin, dass das Folterverbot in Art. 3 EMRK keine Ausnabmen zulässt. Einschränkungen wie nach Art. 15 Abs. 1 EMRK im Falle eines Krieges oder eines anderen öffentlichen Notstandes seien nicht zulässig (Art. 15 Abs. 2 EMRK). Dieses absolute Verbot der Folter und unmenschlicher oder erniedrigender Behandlung oder Bestrafung in den Bestimmungen der Konvention zeige, dass Art. 3 EMRK einen der grundlegendsten Werte der demokratischen Gesellschaften bilde. Die gleiche Regelung könne heute in ähnlichen Bestimmungen anderer internationaler Übereinkünfte gefunden werden. ${ }^{34}$ Der Gerichtshof betont, er sei »sich der immensen Schwierigkeiten, mit denen sich Staaten in modernen Zeiten beim Schutz ihrer Gemeinschaften vor terroristischer Gewalt konfrontiert sehen, durchaus bewusst. Allerdings, selbst unter diesen Umständen verbietet die Konvention in absoluten Begriffen Folter, unmenschliche oder erniedrigende Behandlung oder Strafe, « ${ }^{35}$ unabbängig vom Verhalten des Opfers..$^{36}$ Anders als die meisten anderen konventionsrechtlichen Bestimmungen lasse Art. 3 EMRK keinen Raum für Ausnahmen und erlaube kein Außerkraftsetzen nach Art. 15 Abs. 2 EMRK. ${ }^{37}$

Mit dem Hinweis auf die grundlegenden Werte demokratischer Gesellschaften beruft sich der Gerichtshof zwar auch auf naturrechtliche Begründungen. Diese haben jedoch im normativen Zusammenhang von Art. 3 mit Art. 15 Abs. 2 EMRK ihren positivrechtlichen Ausdruck gefunden. Danach kommt es weder auf die Motive der Verletzer von Art. 3 EMRK an, seien sie auch noch so verständlich und legitim. Die Verletzung des Folterverbots zur Durchsetzung legitimer Ziele zerstört vielmehr die legitime Zielsetzung. Noch kommt es auf das vorangegangene Verhalten des Opfers an. Das absolute Folterverbot markiert vielmehr eine nicht überschreitbare Grenze für Sanktionen, lässt jede Strafe und Behandlung allein wegen des Tabubruchs als unverhältnismäßig erscheinen. Es zeichnet ja gerade den kulturellen Standard einer zivilisierten Nation aus, dass der Zweck nicht absolut gesetzt werden darf, sondern die angewendeten Mittel zur Zweckdurchsetzung in einem angemessenen Verhältnis stehen müssen. Bestimmte Mittel, wie die Folter, sind nicht erlaubt, verletzen allein wegen ihrer Anwendung das Übermaßverbot. Dies gilt selbst bei der Abwehr terroristischer Gefahren. Auch wenn die Gefahr noch so groß, in ihren quantitativen und qualitativen Auswirkungen so bedrohlich ist, die Staaten dürfen zu ihrer Abwehr nicht auf das Mittel der Folter oder anderer unmenschlicher oder erniedrigender Behandlung zurückgreifen. Der Gerichtshof hat deshalb auch folgerichtig den Rechtfertigungsgrund der Terrorismusbekämpfung für die Folteranwendung mit einfachen und klaren Worten zurückgewiesen. ${ }^{38}$

Die Behauptung, auch die Rechtsprechung des Gerichtshofes präge die »Offenheit für eine bilanzierende Gewichtung und Bewertung, « weil dieser »sowohl auf die Intensität des zugefügten Leidens als auch auf den verfolgten $Z_{w e c k}$ « abstelle, ${ }^{39}$ = HRLJ 1990, 335; ECHR, Chabal v. UK, Reports 1996-V, \$79; ECHR, D. v. UK, Reports 1997-III $\int 47$. 
verfehlt die Ratio der dargestellten Rechtsprechung. Wie die Ausführungen zur Terrorismusbekämpfung erweisen, hat es der Gerichtshof vielmehr mit klaren Worten abgelehnt, wegen des besonderen Gewichtes bestimmter politischer Zwecksetzungen irgendwelche Einschränkungen des Folterverbotes zuzulassen. Zwar fordert er für den Begriff der unmenschlichen Behandlung eine Zielgerichtetheit und verlangt er für den Begriff der Folter wie Art. 1 Abs. 1 des Übereinkommens gegen Folter bestimmte Zwecksetzungen, wie etwa die Geständnisoder Aussagenerpressung. ${ }^{40}$ Diese bestimmte Zwecksetzung grenzt jedoch lediglich die Folter von unmenschlicher Behandlung ab, schränkt also nicht den absoluten Schutz des Folterverbotes ein. Darüber hinaus muss regelmäßig eine bestimmte Intention auf seiten des Verletzers festgestellt werden, um unmenschliche Maßnahmen von jenen Maßnahmen abzugrenzen, die notwendigerweise aus der Sicht des Belasteten als unmenschlich empfunden werden, wie etwa die Inhaftierung an sich. ${ }^{41}$ Die Funktion einer derartigen Abgrenzung übernimmt Art. 1 Abs. 1 Satz 2 des Übereinkommens gegen Folter mit dem Begriff der "gesetzlich zulässigen Sanktionen. "Wie ausgeführt, vollzieht der Gerichtshof anhand des Grades des zugefügten Leidens die Abgrenzung zwischen Folter- und unmenschlichen Handlungen; beides gleichermaßen vom absoluten Folterverbot erfasste Gewaltmaßnahmen, gegen die absoluter Schutz gewährleistet wird.

Es ist angesichts der in fünf Jahrzehnten entwickelten, deutschen verfassungsgerichtlichen Rechtsprechung kaum denkbar, dass diese den Schwenk der Schutznormlehre mit vollziehen und offen für »eine bilanzierende Gewichtung und Bewertung « zielgerichtet ausgeübter Schmerzzufügung werden könnte. Andernfalls entstünden schwerwiegende Rechtskonflikte, weil die allgemeinen Regeln des Völkerrechts und damit auch die ius cogens-Regeln lediglich dem einfachen Gesetzesrecht übergeordnet sind, ${ }^{42}$ das Verfassungsrecht diesen also vorgeordnet ist. Maßgebend für die Menschenrechtsbewegung ist ohnehin das Völkerrecht. Auch wenn die deutsche Verfassung für Einschränkungen des Folterverbotes offen werden sollte, bleibt der absolute Schutzcharakter des völkerrechtlichen Folterverbotes strategischer Ausgangspunkt für die Menschenrechtsbewegung. Ermutigend ist insoweit auch, dass bei aller Ambivalenz, welche die Rechtsprechung des US-Supreme Court kennzeichnet, dieser - wohl alarmiert durch das »Foltermemorandum « des Verteidigungsministeriums - mit Leidenschaft gegen die Auffassung der Bush-Administration Position bezogen hat, es könnten mit Berufung auf den »war on terrorism « rechtsfreie, die Folter begünstigende Räume zugelassen werden. ${ }^{43}$ In diesem Gesichtspunkt kann diese Rechtsprechung durchaus als Bekräftigung des notstandsfesten Charakters des Folterverbotes verstanden werden.

\section{Der Legitimationsgrund "Historische Unrechtserfabrungen"}

Allein der positivrechtliche Ansatz greift für die Entwicklung einer menschenrechtlichen Strategie zu kurz. Terroristische Bedrohungen schaffen permanente Unruhe, massenhafte Unsicherheitsgefühle, rufen eine allgemeine Verunsicherung hervor, die für jede Ordnung und ihre Institutionen sowie deren sinnstiftende 
können. Sich wiederholende Anschläge von der Qualität des 11. September 2001 in den Vereinigten Staaten und vom 11. März 2004 in Madrid könnten sehr schnell einen "permanenten Ausnabmezustand " und eine "fürsorgliche Despotie"im Namen nationaler Sicherheit (Richard Rorty) hervorbringen. Die Vision einer offenen Gesellschaft wäre nur noch ein Topos antiquarischer Bücher. Die Menschenrechtsbewegung muss deshalb nach Begründungen suchen, die dem positivrechtlichen Ansatz vorgelagert sind. Dies sind insbesondere und an erster Stelle die gesicherten historischen kollektiven wie individuellen Unrechtserfahrungen, ${ }^{45}$ die mit der Folteranwendung verbunden sind und die belegen, dass jede Einschränkung des absoluten Verbotes nicht mehr regulierbare gesellschaftliche und politische Auswirkungen hat und zur nicht wieder heilbaren seelischen Zerstörung des Folteropfers wie des Folterers führt. Gerade die Menschenrechtsbewegung kann aufgrund ihrer Erfahrungen mit der Folterpraxis für diese eskalierende Dynamik der Folter überzeugungskräftige Strategien entwickeln.

So weist etwa amnesty international im ersten Folterbericht auf die historischen Erfahrungen mit der Folter hin, die ein "praktisches Argument « gegen ihre Anwendung liefere. Die Geschichte zeige, dass die Folter niemals begrenzt sei. Werde die Folter einmal erlaubt, würden bald die Grenzen ihrer vermeintlichen Regulierung überschritten, und neue Kapitel würden den Annalen menschlicher Grausamkeit und Leiden hinzugefügt. Dem zugelassenen Einzelfall folge der nächste Einzelfall, die Folteranwendung werde zur Praxis und schließlich zur Institution. Im Alten Rom sei die Folter zunächst gegen jene angewendet worden, die gegen die souveräne Macht handelten. Zunächst zurückhaltend praktiziert, habe sie sich im gleichen Maße ausgeweitet, wie der despotische Charakter der Macht des Souveräns zugenommen habe. »Sobald die Folter erst einmal erlaubt ist - z. B. in einer extremen Situation, wie im Beispiel mit der Bombe - dann ist es nur logisch, sie auf Leute anzuwenden, die Bomben legen könnten oder auf Leute die daran denken könnten, Bomben zu legen, oder auf Leute, die die Leute, die ans Bombenlegen denken könnten, verteidigen. Das Beispiel Algerien ist ein klassischer Fall. Die Folter begann mit gewissen Einschränkungen, und dann weitete sie sich aus zu einer Orgie der Brutalität; ihre Opfer waren zuerst Einheimische, dann griff sie auf Frankreich über. Sie war wirksam als Waffe im Kampf, und die Franzosen gewannen die Schlachten, aber sie verloren den Krieg. Der Krebs ist eine passende Metapher für die Folter und ihre Ausbreitung im gesellschaftlichen Organismus. Die Praxis der Folter kann nicht vom Rest der Gesellschaft getrennt werden; sie hat ihre Konsequenzen, sie erniedrigt diejenigen, die sie anwenden, die von ihr profitieren, und sie ist der denkbar eklatanteste Widerspruch zur Gerechtigkeit, eben jenem Ideal, auf das der Staat seine Autorität zu gründen bestrebt ist. « ${ }^{46}$

Die historischen Unrechtserfahrungen beziehen sich insbesondere auf politische Systeme, in denen die Folter systematisch und in institutionalisierter Form praktiziert wurde. Wegen der eskalierenden Dynamik der Folter und auch aus konkretem Anlass erregen jedoch insbesondere die politischen Entstehungsbedingungen von Folter das Interesse. Wohin führt die Zulassung von Ausnahmen vom Folterverbot in demokratischen Rechtsstaaten? Was sind die Folgen für diese Systeme, wenn den Ausnahmen nicht widersprochen wird? Wie kann Widerstand unter Bedingungen der Folterbedrohung organisiert werden? In der Literatur 
werden zwei Anwendungskontexte von Folter unterschieden: Die situationsspezifische Folter im Fall einer Bedrohung der nationalen Sicherheit durch Terrorismus, Guerilla und Aufstände gehe zumeist einher mit der Außerkraftsetzung einiger systemstruktureller und rechtlicher Sicherungen. Exekutive Befugnisse würden verstärkt, Grundrechte eingeschränkt und Schutzvorkehrungen gegen den Missbrauch von Gewalt gegenüber Inhaftierten aufgehoben. Davon wird die institutionalisierte Folter als Bestandteil systematischer Unterdrückung friedlicher politischer Opposition, ethnischer und religiöser Minderheiten oder zur allgemeinen Terrorisierung der Bevölkerung unterschieden. Der Übergang von der ersten zur zweiten Kategorie erfolge häufig durch einen Putsch. Die Bekämpfung staatlicher Duldung der Folter in einer funktionierenden rechtsstaatlichen pluralistischen Demokratie könne sich auf funktionierende Strukturen der Herrschaftsbegrenzung stützen. Um institutionalisierte Folter einzudämmen, bedürfe es dagegen der strukturellen Sicherung von Herrschaftsbegrenzung. ${ }^{47}$

Diese Unterscheidung ist fragwürdig, weil sie übersieht, dass die Zulassung situationsspezifischer Folter auf Dauer Strukturen der Herrschaftsbegrenzung von innen heraus zerstört und deshalb den gesellschaftlichen Widerstand gegen staatliche Folterpraxis erschwert und schließlich unmöglich macht. Darüber hinaus findet die situationsspezifische Folter nicht erst unter den Bedingungen des Ausnahmezustands statt, sondern - wie die Fälle Selmouni ${ }^{48}$ und Daschner erweisen - kann sie bedeutend früher einsetzen, unter völlig normalen Verhältnissen im demokratischen Rechtsstaat. Damit die Menschenrechtsbewegung - gemünzt auf derartige Situationen - überzeugungskräftige Strategien entwickeln kann, sind Studien über die Veränderungen der gesellschaftlichen und staatlichen Institutionen in funktionierenden demokratischen Rechtsstaaten erforderlich, in denen Verletzungen des Folterverbotes festgestellt wurden. Die griechischen, chilenischen und verschiedenen türkischen Juntasysteme, in denen die Folter vom ersten Tag der Herrschaft an integrierter Bestandteil des Staatsapparates zur Unterdrückung der Opposition und zur allgemeinen Terrorisierung gewesen war, bezeichnen dagegen den Typus des schlagartigen Übergangs nach einem Militärputsch. Verhüllt die Folter wie in derartigen Systemen nicht mehr ihr Gesicht hinter moralisch hochgreifenden Legitimationsformeln, offenbart sie vielmehr in unverfälschter Deutlichkeit ihre barbarische Fratze, besteht Einigkeit über ihre Unzulässigkeit. Die Sensibilität für den zerstörerischen Charakter der Folter muss jedoch bedeutend früher ansetzen: beim ersten bekannt werdenden Folterfall im demokratischen Rechtsstaat.

Festzuhalten ist, dass bereits mit der Zulassung der situationsbezogenen Folter im funktionierenden Verfassungsstaat der Weg für die Umwandlung in ein System institutionalisierter Folter vorgezeichnet wird. Die Vorstellung, dass sich Folter rechtsstaatlich »domestizieren « ließe, ist in sich widersprüchlich. Die positivistische Vorstellung einer » rechtsstaatlich eingebundenen « Folter ist nicht nur in der Theorie ein Unding, sondern auch in der Praxis unmöglich. Der Staat, der Folter erlaubt, eröffnet den Sicherheitsorganen Ermessensspielräume, die eine Grauzone hervorbringen, in der Folter zur Gefahrenabwehr fortan erlaubt ist. Damit sind Dammbrüche programmiert. Zum anderen unterminiert der die Folter zulassende Rechtsstaat seine eigene Legitimationsbasis, die er folglich schwerlich zur Begrenzung der einmal eröffneten Folterpraxis geltend machen kann. ${ }^{49}$ Wenn die Unterscheidung zwischen situationsspezifischer und institutionalisierter Folter auch 
problematisch ist, bekräftigt sie andererseits doch die These, dass die Zulassung von Ausnahmen vom absoluten Folterverbot eine unkontrollierbare eskalierende Repressionsspirale in Bewegung setzt. Denn die Unterscheidung ist eine strategische, macht deutlich, dass Folterpraxis in unterschiedlichen Kontexten vorkommt und ihre Bekämpfung deshalb unterschiedliche Gegenstrategien erfordert. Es wird bei dieser Unterscheidung aber die evidente Tatsache vorausgesetzt, dass fehlende Mobilisierung gegen situationsbezogene Folter diese Praxis institutionalisiert. Die gesellschaftliche Duldung derartiger Folterpraxis öffnet mithin der totalen Herrschaft der Folter das Tor und zersetzt zunächst den Staat von innen; anschließend richtet sich das aufgestaute gesellschaftliche und staatliche Aggressionspotenzial nach außen gegen andere Staaten. Nur durch einen wirksamen gesellschaftlichen Kampf gegen jeden bekannt werdenden Folterfall kann die in Gang gesetzte Eskalationsspirale umgedreht werden. Dazu gehören nicht nur entschiedener Widerstand gegen Relativierungen des Folterverbots in der Praxis wie im Falle Daschner, sondern auch die Entwicklung überzeugungskräftiger verfassungsrechtlicher Positionen gegen die Schutznormlehre. Was vor allem ernsthafte Besorgnis erregt, ist die verfassungsrechtliche Legitimierung der von Praktikern erhobenen Forderung nach Relativierung des Folterverbotes. Gegenüber einem derart praktisch-theoretisch aufgeladenen Diskurs reicht es nicht aus, lediglich den konkreten Einzelfall aufzugreifen. Vielmehr muss die Menschenrechtsbewegung die $\mathrm{Ge}-$ sellschaft der Verfassungsinterpreten « mobilisieren, um den verfassungsrechtlichen und -politischen Konsens, auf dem auch die verfassungsrechtliche und internationale Rechtsprechung beruht, für die uneingeschränkte Achtung des Folterverbotes zu bewahren und zu stärken.

Die Befürworter der »Rettungsfolter « verkennen die Aufgabe des Rechts. Werden keine Anstrengungen gegen situationsbezogene Folterfälle unternommen, ist der Siegeszug totaler Herrschaft und damit der Verneinung der Herrschaft des Rechts nicht mehr aufzuhalten. Der unbeirrbare Einsatz gegen jeden Foltervorgang ist danach notwendig, um die zivilgesellschaftlichen Strukturen als soziale Basis der Herrschaftsbegrenzung zu bewahren und zu stärken. Historisch gesicherte Erfahrungen belegen, dass jegliche Einschränkung des Folterverbotes am Ende zivilgesellschaftliche Strukturen zersetzt und totalitäre Herrschaftsformen hervorbringt. Deshalb muss das Rechtssystem demokratischer Rechtsstaaten eindeutig sein: Es darf die Folteranwendung auch unter bestimmten Ausnahmebedingungen nicht zulassen, muss jene, die das Folterverbot verletzen, strafrechtlich und disziplinarisch zur Verantwortung ziehen und rechtliche und politische Strukturen gewährleisten, die die offene pluralistische gesellschaftliche Auseinandersetzung als Grundbedingung herrschaftskritischer Opposition und als Grundlage eines diskursiven Rechtssystems ermöglicht.

\section{Die Verflüchtigung historischer Unrechtserfahrungen im "Präventionsstaat"}

Alle historische Erfahrung spricht danach gegen die Zulassung von Ausnahmen vom Folterverbot. Deshalb ist der unbeirrbare Widerspruch gegen jeden Einzelfall von Folter bereits im demokratischen Rechtsstaat ein zwingendes politisches und rechtliches Gebot. Neuartige terroristische Bedrohungen haben jedoch eine komplexe gesellschaftliche und politische Gemengelage hervorgebracht. Es besteht die ernsthafte Gefahr, dass die beschleunigte Wandlung der demokratischen Verfassungsstaaten zum Modell des "Präventionsstaates" die bisherige, gesellschaftlich wirksame Kraft historischer Unrechtserfahrungen entscheidend schwächen wird. 
Zutreffend weist der Vizepräsident des Bundesverfassungsgerichtes auf eine "präventionsorientierte" Gesellschaft hin, die nicht mehr "normfolgenorientiert" ist ${ }^{50}$ also die in Schutznormen verdichteten historischen Unrechtserfahrungen nicht mehr als stete Mahnung für das gesellschaftliche Miteinander begreift. Es ist die Funktionslogik dieses Staatsmodells, welche die Befürchtung aufkommen lässt, dass unter den Bedingungen eines vorrangig auf die »Gefahrenabwehr « gerichteten Staates die Zulassung von Ausnahmen vom Folterverbot unumkehrbare Auswirkungen haben und die demokratische Substanz der Verfassungsstaaten von innen heraus zerfressen wird. Es liegt in der Logik der Schutznormlehre, dem verfassungsrechtlichen Schwert des Präventionsstaates, dass dieser Staat das »absolute Folterverbot « nicht dulden kann. Denn er verspricht den Bürgern ein „Grundrecht auf Sicherheit «. Zu seiner Logik gehört die Maßlosigkeit, weil Grenzenlosigkeit eines nie erreichbaren Ideals. Dieses Ideal heißt Sicherheit. Sicherheit ist nicht mehr die »Gewissheit der gesetzmäßigen Freiheit « (Wilhelm von Humboldt), sondern Sicherheit meint jetzt »die Zusage einer prinzipiell unbegrenzten, nie endenden staatlichen Aktivität zum Schutze der Bürgers vor sozialen, technikund umweltbedingten oder auch kriminellen Risiken und Gefahren. « ${ }^{51}$ Es liegt in der Logik des Präventionshandelns, dass es nicht mehr nur an »Gefahr«, an generelle potenzielle Kriminalität, nicht mehr an »Verdacht« anknüpft, sondern im Verhältnis Staat-Bürger eine generelle Umkehr der Beweislast stattfindet. Weil das »Risiko« immer und überall existiert, wird es zur Normalität, die Nichtgefährlichkeit bildet dann die Ausnahme, die der Bürger für seine Person beweisen muss. ${ }^{52}$

Trotz der unterschiedlichen Anknüpfungspunkte der Kindesentführung einerseits und terroristischer Bedrohungen andererseits fügen sich die Diskussionen um die Zulässigkeit von Folter in den gemeinsamen Kontext des Bestrebens nach mehr Sicherheit ein. In beiden Fällen geht es um Folteranwendung zur Abwendung vermuteter bevorstehender Angriffe und Verbrechen. Terroristische Aktionen wie Kindesentführungen treffen ein Gefühl unabwägbarer, persönlicher Gefährdung in der Bevölkerung. In beiden Fällen scheinen die Bedrohungen zufällig, wenig greifbar, können sie jeden treffen. Aktuelle Ereignisse werden daher emotionalisiert in einem Szenario allgemeiner Bedrohung reflektiert, das die Forderung nach effektiver polizeilicher Arbeit nahe legt. ${ }^{33}$ Sicherheit wird unter diesen Voraussetzungen ein selbstevidenter öffentlicher Belang, der keiner normativen verfassungsrechtlichen Herleitung mehr bedarf. Die Abwägung von Freiheit und Sicherheit wird einseitig. Rechtlich wird diese Entwicklung dadurch begründet, dass der polizeirechtliche Gefahrenbegriff von individuellen Handlungen entkoppelt wird. Denn beim internationalen Terrorismus lassen sich Gefahren nicht mehr tatbestandlich bestimmen und individualisieren, da sie von losen Netzwerken ausgehen. »Proaktives Polizeihandeln« ist gefordert. Die Konturen solcher Aktionen werden aber nicht durch raum-zeitlich bestimmbare Schadenswahrscheinlichkeiten bestimmt, vielmehr bleiben sie unbestimmt, so unbestimmt und unberechenbar wie die Risiken, deren Verwirklichung sie verhüten sollen. Es entfällt die Möglichkeit, präventives Staatshandeln als »Gegenmaßnahme an vorangegangenes Tun der Straftäter zu orientieren, zu individualisieren und damit zu dosieren. ${ }^{54}$ Die Freiheit des Einzelnen wird in dieser Konstellation nicht mehr indi- 
die Freiheit der Gesellschaft bedroht ist, muss er mögliche, auch an weit unterhalb konkreter Verdachtsmomente anknüpfende Einschränkungen seiner individuellen Freiheit hinnehmen, sofern sie dem Ziel der Sicherung der gesellschaftlichen Freiheit dienen. Damit wird die individuelle Freibeit zu einer Freibeit unter Gesellschaftsvorbebalt. ${ }^{55}$ Sicherheit zielt nicht mehr auf Rechtssubjekte als Verpflichtete, und auch auf der Berechtigtenseite wird Sicherheit subjektlos, da sie auf die Gesellschaft bezogen wird.

Wird diese Entwicklung des Präventionsstaates zu Ende gedacht, gewinnen die Gefahren, die mit der Zulassung von Ausnahmen vom Folterverbot ausgelöst werden könnten, schärfere Konturen: Die Schutznormlehre knüpft für die Gefahrenabwehr durch »Rettungsfolter « an den polizeirechtlichen Störerbegriff und an die »Pflicht zur Gefahrenbeseitigung « an. ${ }^{56} \mathrm{Da}$ aber im Präventionsstaat der Störerbegriff zunehmend entindividualisiert und deshalb auch die Verdachtsschwelle zunehmend herabgesetzt wird, andererseits nach der Schutznormlehre grundsätzlich alle geeigneten und verfügbaren Mittel und somit prinzipiell auch die Folter zugelassen wird und diese bei besonders großen Gefährdungen auch angewendet werden soll, wird die denkbar schärfste Form der Menschenrechtsverletzung von individuell zurechenbarem Störerverhalten vollständig entkoppelt. Zu Recht beklagt der Vizepräsident des Bundesverfassungsgerichtes, dass die Gesellschaft in einer gefährlichen Situation in der Wahl der Mittel frei sein, jede Möglichkeit, Rechtsgutverletzungen zu verhindern, ergreifen wolle, egal was es koste. ${ }^{57}$ Die Schutznormlehre legt dem Betroffenen die Beweislast dafür auf, dass er zur Gefahrenabwehr nichts beitragen kann. Im Zweifel muss er also die Folter erdulden. ${ }^{58}$ Historische, mit der Folteranwendung verbundene Unrechtserfahrungen verlieren ihre bisherige gesellschaftlich wirksame Kraft, können gegenüber einem gefestigten, durch die Schutznormlehre geprägten gesellschaftlichen und verfassungsrechtlichen Konsens nicht mehr durchdringen. Mit einer derart verfassungsrechtlichen Grundausrüstung ausgestattet, löst die verfassungsrechtlich legitimierte Zulassung eng begrenzter Ausnahmen vom Folterverbot unter den Bedingungen des Präventionsstaates unverzüglich einen Flächenbrand aus.

\section{Gegen die »Bilanzierung« des Unverfügbaren}

Die "menschliche Würde" ist einer "bilanzierenden Gewichtung und Bewertung "59 nicht zugänglich. Die buchhalterisch-kaufmännische Verwertung eines unverfügbaren, jeder menschlichen Verfügung entzogenen Wertes ist das vielleicht erschreckendste Moment am neuen verfassungsrechtlichen Diskurs. Es liegt in dem auf Universalität gerichteten Anspruch der westeuropäischen politischen Philosophie seit dem Zeitalter des »Vernunftrechts «, die »Natur« des Menschen allgemeingültig, abstrabiert von allen empirischen, historischen, gesellschaftlichen und politischen Bedingungen bestimmen zu wollen. Der Gedanke der »natürlichen«, »eingeborenen « und »unveräußerlichen« Rechte, die das Individuum in den staatlichen Verband einbringt, der zwar in der praktischen Wirklichkeit vielfach gebrochen, doch als das bewegende Moment der Geschichte der Menschenrechte 
unverändert und unabdingbar Geltung einfordert, hat aber die »Begrenzung jeder staatlichen Herrschaft durch Recht, die Bindung der staatlichen Macht mindestens an jene Rechte, zur unausweichlichen Konsequenz. Dies ist die Idee des modernen Rechtsstaates. Wie die Grenze zwischen Bürgerfreiheit und Staatsgewalt im Einzelnen auch immer verlaufen mag: der Leviathan ist durch Recht und Gesetz gebunden, nie darf er den Menschen in seiner Totalität ergreifen. $\aleph^{60} \mathrm{Nicht}$ schon die Grundrechte, wohl aber die Menschenrechte setzen notwendigerweise voraus, dass es im Rechtsverhältnis zwischen Staat und Individuum etwas gibt, was nicht erst vom Gesetzgeber gewährt wird, sondern von ihm als vorgängig anerkannt und als nicht verfügbar respektiert werden soll. Danach kommt dem Menschen eine Eigenständigkeit gegenüber allen Weltbezügen, in die er verflochten ist, zu. Er ist also nicht nur Ensemble der gesellschaftlichen Verhältnisse, sondern er kann sich noch einmal gegenüber der Gesamtheit der Weltverhältnisse, die er ist, verhalten. ${ }^{61}$ Der Mensch ist nicht das Wesen der Willkür, sondern der Vernunft, und zwar nicht einer fremden, von außen kommenden Vernunft des Leibes oder gesellschaftlicher Gesetzmäßigkeiten, sondern seiner eigenen, von ihm selbst gesetzten Vernunft. ${ }^{62}$

Aus dieser Idee eines sich selbst bestimmenden, nicht von außen verfügbaren Individuums schöpfen die Menschenrechte ihre revolutionäre Sprengkraft, bauen die Geschichte der Menschenrechte und die Geschichte moderner gewaltenteilender und grundrechtssichernder Rechtsstaaten auf. Es ist die gewissenhafte Umsetzung der Idee der menschlichen Selbstbestimmung, die den "normativen Ursprung grundrechtlicher Garantien « bezeichnet »und deren unbedingter - in der Idee der Würde eines jeden Menschen begründeter Verbindlichkeit. « ${ }^{63}$ Die verfassungsrechtliche Ordnung dient der Autonomie des Individuums. Sie setzt sie zudem voraus, indem sie den Menschen zum Legitimationssubjekt der Verfassung erklärt. Die Verfassung schützt die Autonomie des Menschen daher nicht nur aus Respekt vor seiner Individualität, sondern auch als Voraussetzung eines demokratischen Gemeinwesens und als verfassungsrechtliches Legitimationssubjekt. ${ }^{64} \mathrm{Die}$ Folter hingegen erfasst den Menschen total, kehrt sein Innerstes mit unbedingtem Zwang nach außen. Der Betroffene wird zum total verfügbaren Objekt gemacht, das keinerlei Möglichkeiten mehr zur Wahrnehmung eigener Rechte hat. ${ }^{65}$ Die Folter zerreißt die auf Vernunft und Achtung dem Gegenüber aufbauenden Kommunikationsstrukturen, ersetzt Sprache durch auf Erniedrigung, Unterwerfung und Vernichtung zielende unbedingte Gewalt und zerstört damit alle Voraussetzungen einer nur als diskursive Kommunikationsgemeinschaft vorstellbaren, staatlich verfassten Gesellschaft.

Anders als die Tötung ist die Folter ein Mittel zur fortgesetzten Verfügbarmachung der Psyche des Gefolterten, das über die Unschädlichmachung weit hinausgehend die innere Unterwerfung dauerhaft belegen will. ${ }^{66}$ Deshalb verfehlt der Hinweis auf den »finalen Rettungsschuss « zur Rechtfertigung der »Rettungsfolter « das entscheidende Merkmal der Folter. Bereits der normativ geschulte Blick belegt die Fragwürdigkeit dieses Vergleichs, denn die Tötung ist unter bestimmten Voraussetzungen erlaubt (vgl. Art. 2 Abs. 2 EMRK). Die Brechung des Willens 
Erfahrungen deuten darauf hin, dass die Folter für diejenigen, die sie anwenden oder befürworten, immer auch zumindest mehr als ein Mittel zur Wahrheitsfindung ist. ${ }^{67}$ Der » Rettungsfolter « wohnt damit stets auch ein zerstörerischer Überschuss inne, der über die bloß präventiv motivierte Aussagenerzwingung hinausgeht und dazu führt, dass zur Brechung des Willens des Aussageunwilligen dessen Psyche gezielt zerstört wird. Sie zielt mehr als alles andere direkt auf den Körper des Menschen und dient dazu, diesen Körper und die Persönlichkeit des Gefolterten zu zerstören. Folter ist immer, auch wenn sie reglementiert ist, in extremer Weise Machtausübung des Folternden oder seiner Auftraggeber gegenüber dem Gefolterten. Dadurch wird Herrschaft hergestellt, inszeniert und aufrechterhalten. Durch ihre abschreckende Wirkung trifft die Folter dabei nicht nur den Gefolterten, sondern hat insgesamt zeichenhaften Charakter. ${ }^{68}$ Die Folter überschreitet mithin jene Grenze, die dem modernen demokratischen Rechtsstaat um seiner selbst willen gesetzt ist und aus der er zugleich seine Legitimation erfährt. Mit dem Tabubruch zerstört der Staat sich also selbst. Es steht damit alles auf dem Spiel, wenn Ausnahmen vom Folterverbot zugelassen werden. So verständlich der Drang ist, Leben unter allen Umständen und unter Anwendung aller verfügbaren technischen Mittel zu retten, die Anwendung eines den Menschen in seiner Totalität erfassenden Mittels zerstört die Grundlagen des Staates. Man kann verstehen, dass der zum Schutz von Gefährdeten berufene Polizeibeamte in der zerreißenden Anspannung der konkreten Situation die Nerven verliert. Doch darf ein derart verstehbarer Fehler unter der Herrschaft des Rechts nicht positivrechtlich erlaubt und sogar angeordnet werden, wie dies die Befürworter der »Rettungsfolter « fordern. Im Fall Daschner erfolgt die Anweisung zur Folter indes vorbedacht und sollte eigens zu deren Ausführung ein Spezialist eingeflogen werden.

Grund- und Menschenrechten eignet der Bezug auf die voraussetzungslose Würde der Person. Gegen sie verstieße man, wenn man die Rechte nur dem zukommen ließe, der sich durch Pflichterfüllung gegen die Gemeinschaft als würdig erwiesen hat. Räumt das Gemeinwesen dem Menschen als Menschen Rechte ein, so muss es in Kauf nehmen, dass die menschenrechtliche Komponente der Grundrechte eine direkte Parallelität zwischen Rechten und Pflichten ausschließt. Nur wo die Gesellschaftsordnung eine Interessenidentität zwischen Individuen und Gemeinschaft - wie in gleichgeschalteten totalitären Gemeinschaften - voraussetzt, ist eine Symmetrie von Rechten und Pflichten möglich. Wo dies nicht der Fall ist, sie aber dennoch erzwungen wird, geht die Tiefendimension der Menschenwürde verloren. ${ }^{69}$ Daher verfehlt die Ansicht, dass die Misshandlung nur dazu dienen soll, den Erpresser in dem ihm zustehenden Rechtsraum zurückzudrängen, ${ }^{70}$ weil - kantisch gesprochen - das Recht gewahrt bleiben müsse, ${ }^{71}$ das Wesen der Menschenwürde und der Sittenlehre Kants. Denn Kants kategorischer Imperativ wird durch Folteranwendung in sein Gegenteil verkehrt. Die Funktionalisierung des Menschen ist vollständig, und sein Anspruch auf Achtung als Selbstzweck wird restlos negiert. $^{72}$

Nicht beantworten können die Befürworter der »Rettungsfolter « die Frage, aus welchen Gründen ein Unschuldiger, der über die erforderlichen Informationen verfügt, nicht gefoltert werden darf. Die Logik der Gefahrenabwehr kann auch 
einen Journalisten nicht verschonen, der sich auf die Zusicherung der vertraulichen Behandlung erhaltener Informationen beruft, die er bei einem Interview mit einem gesuchten terroristischen Straftäter erlangt hat, dessen Aufenthaltsort er aber aus legitimen Gründen nicht preisgeben möchte. Ebensowenig kann sie Rücksicht nehmen auf den Rechtsanwalt des als gefährlich eingeschätzten Mandaten, der im anwaltlichen Beratungsgespräch mutmaßlich Wissen über eine unmittelbar bevorstehende Gefahr erlangt hat. Sie kann auch den Zufallszeugen eines entscheidenden Geschehens nicht verschonen, der aus Angst vor Vergeltung schweigt. Da für den technokratisch »bilanzierenden «, nüchternen Rechner vorrangig der Umfang und die Größe der abzuwehrenden Gefahr ins Gewicht fällt und allein deshalb unverbrüchliche Werte aufgelöst und in die Bilanz eingestellt werden, ist nach der Logik der Schutznormlehre der polizeirechtliche Störerbegriff und die »Pflicht zur Gefahrenbeseitigung « entscheidend, ${ }^{73}$ sodass nach dieser Lehre kein Grund ersichtlich ist, warum der Unschuldige, der zur Gefahrenabwehr beitragen kann, nicht gefoltert werden dürfte. Dass die Schutzwirkung des absoluten Folterverbotes unabhängig vom Verhalten des Opfers eingreift, ${ }^{74}$ ist für die präventionsorientierte Schutznormlehre unerheblich. Vielmehr fragt sie gar nicht mehr nach dem individuellen Verhalten des Opfers, sondern allein danach, welchen Beitrag dieses zur Gefahrenabwehr möglicherweise leisten kann, und erlaubt die Folter, um im Weigerungsfall diesen Beitrag zu erzwingen. Der Einzelne wird im bilanzierenden Rechenwerk des Präventionsstaates mechanistisch auf seinen gesellschaftlichen Nutzen reduziert.

Rechnungen, die das durch die Folter Erreichbare, die Rettung des entführten Kindes oder die Entschärfung einer eine ganze Stadt bedrohenden Bombe, gegen die möglichen Schädigungen des Opfers aufwiegen, also Würde gegen Würde aufwiegen, ${ }^{75}$ liegt ein entscheidender Fehler zugrunde, weil man die hier angegebenen Größen nicht aufeinander beziehen kann. Man kann die menschliche Würde wegen ihrer Unverfügbarkeit nicht mit anderen Größen, etwa mit der Würde der bedrohten Personen, aufwiegen, weil beide Größen je für sich unverfügbar, wenn man so will »bilanzierungsfeindlich « sind. Man muss wegen der historischen Unrechtserfahrungen und der voraussehbaren Folgen langfristig denken und notfalls in Situationen »auch hohe Interessen, hohe Rechtsgüter opfern, um nicht langfristig die Zivilität, die Anständigkeit, das Leben-Können innerhalb einer Rechtsordnung zu gefährden und zu zerstören. $\ll^{76}$ Mit der Folteranwendung werden nichtrücknehmbare Tatsachen geschaffen, welche Geschädigten wie Schuldigen mit jeweils ihrem Anteil, der aus der sie zunächst verbindenden Situation des Folteraktes hervorgeht, für immer allein lassen. Dieser Anteil des Nichtrücknehmbaren an humaner Selbstverstümmelung, der Opfer wie Peiniger stigmatisiert, vernichtet bereits im Ansatz jeden möglichen Rechtfertigungsversuch der Folter. $^{77}$

Im demokratischen Rechtsstaat darf nur dort Zwang ausgeübt werden, wo Autonomie eingeschränkt wird, die Handlung des einen mit der des anderen nicht mehr »nach einem allgemeinen Gesetz der Freiheit zusammen vereinigt werden kann « (Kant). Diese Einschränkung von Zwangsmaßnahmen auf den rechtlichen Zwang im Interesse der Autonomie ist der wesentliche Inhalt der Freiheitsrechte. ${ }^{78}$ Dabei 
macht bereits die Funktion der staatlichen Zwangsausübung, das ist die Wahrung der Autonomie des Einzelnen, bewusst, dass ihr eine unüberschreitbare Grenze durch die Autonomie des Einzelnen gesetzt ist. Daher verfehlen Rechnungen wie »Würde gegen Würde, Leben gegen Leben «79 das Wesen der polizeilichen Zwangsanwendung im demokratischen Rechtsstaat. Zwar sind die staatlichen Organe zu einem effektiven Schutz des menschlichen Lebens verpflichtet. Sie verbietet nicht nur unmittelbare staatliche Eingriffe in das Leben, sondern gebietet dem Staat auch, sich schützend und fördernd vor dieses Leben zu stellen, das heißt vor allem, es auch vor rechtswidrigen Eingriffen von seiten anderer zu bewahren. Sie befinden jedoch darüber, welche Schutzmaßnahmen zweckdienlich und geboten sind. Ihre Freiheit der Wahl der Mittel zum Schutz des Lebens kann sich in besonders gelagerten Fällen auch auf die Wahl eines bestimmten Mittels verengen, wenn ein effektiver Lebensschutz auf andere Weise nicht zu erreichen ist. ${ }^{80}$ Dabei stellt aber der absolute Achtungsanspruch der Menschenwürdegarantie eine unabdingbare Grenze bei der grundsätzlichen Wahlfreiheit der Mittel dar, deren Verletzung trotz der besonders zentralen Aufgabe des Staates, menschliches Leben zu schützen, nicht überschritten werden darf.

\section{Schlussfolgerungen}

Die Schutznormlehre und die Daschner-Debatte haben zentrale, bislang für unumstößlich erachtete Gewissheiten erschüttert. Angesichts des Tempos der technologischen Entwicklung sowie auch der Abfolge der politischen Veränderungen und der politischen Brüche seit 1989/1991 ist der Tabubruch grundsätzlich kein Übel. Im Gegenteil, er zwingt uns, bisherige, als gesichert vorgestellte Grundannahmen im Lichte neuartiger Herausforderungen zu überprüfen, gegebenenfalls zu ändern und den neuen Verhältnissen anzupassen. Das absolute Folterverbot als sinnfälliger Ausdruck der unantastbaren Menschenwürde genießt jedoch Artenschutz, jedenfalls für jene, deren Menschenbild auf der Vision einer Welt freier Menschen in freien Gesellschaften in demokratisch verfassten Staaten beruht und die nicht wollen, dass der Mensch den Menschen vernichtet. Wer das absolute Folterverbot einschränken will, will ein anderes Menschenbild, eine andere Gattung und eine andere Gesellschaftsordnung und sollte den Mut besitzen, dies auch zuzugeben, oder seine Meinung überprüfen.

Allerdings wird die Menschenrechtsbewegung durch die herauf gezogene Debatte gezwungen, ihre Begründungsmuster für das absolute Folterverbot zu überprüfen und für die Öffentlichkeit plausibel und nachvollziehbar zu machen, warum das unbeirrbare Festhalten am absoluten Folterverbot so überaus zwingend für das friedliche Miteinanderleben der Menschen und der Menschheit insgesamt ist. Sie muss auch dann standfest bleiben, wenn nach spektakulären Verbrechen in der nachfolgenden erhitzten Debatte die Verletzung des Folterverbotes als einziges effektives Mittel zur Lebensrettung eines Kindes oder gar einer unbestimmten Vielzahl von Menschen als unausweichlich dargestellt wird und der aufgebrachte kollektive Zorn sich auf jene entlädt, die gute Gründe dafür haben, selbst in derartigen Situationen an ihren Überzeugungen festzuhalten. Die durch Daschner 2002/2003 entzündete Debatte ist so neu nicht. Bereits im Folterbericht von 1973 
behandelt amnesty international das Beispiel mit der Bombe im Jumbo Jet ${ }^{81}$ und wird in einem 1976 heraus gegebenen Sammelband das Beispiel des entführten Kindes erörtert. ${ }^{82} \mathrm{Neu}$ ist, dass, anders als nach der Veröffentlichung der Monographie von Ernst Albrecht im Jahre 1976, Meinungsumfragen für die Zulässigkeit der » Rettungsfolter « durchgeführt werden, ein hoher Justizbeamter sich bei Misshandlungen durch Staatsorgane auf die Frage nach der strafrechtlichen Verantwortung in diffusen Windungen verliert und dass in dem führenden Standardkommentar der Verfassungsrechtler die Schutznormlehre zur Legitimation der »Rettungsfolter « »verfassungsfest « gemacht werden soll und zugleich bisherige Grundannahmen über unverbrüchliche und unantastbare Werte unserer Verfassungsordnung durch kaufmännisch bilanzierendes Rechnen aufgelöst werden. 